\title{
The Effects of Various Basis Image Priors on MR Image MAP Restoration
}

\author{
S. Garnier \\ G. Bilbro \\ J. Gault \\ W. Snyder
}

Center for Communications and Signal Processing Department of Electrical and Computer Engineering North Carolina State University

$$
\begin{gathered}
\text { TR-94/10 } \\
\text { April } 1994
\end{gathered}
$$




\title{
The Effects of Various Basis Image Priors on MR Image MAP Restoration
}

\author{
Stephen J. Garnier and Griff L. Bilbro \\ Department of Electrical and Computer Engineering \\ North Carolina State University \\ Raleigh, NC 27695-7911 \\ James W. Gault \\ U. S. Army Research Office \\ Research Triangle Park, NC \\ Wesley E. Snyder \\ Department of Radiology, Bowman-Gray School of Medicine \\ Wake Forest University, NC \\ Accepted for publication by JMIV; modifications are underway.
}

\section{ABSTRACT}

We extend a previously reported technique for Magnetic Resonance Image (MRI) restoration, using a physical model (spin equation) and corresponding basis images. We determine the basis images (proton density and nuclear relaxation times) from the MRI data and use them to obtain excellent restorations.

Magnetic Resonance Images depend nonlinearly on proton density, $\rho$, two nuclear relaxation times, $T_{1}$ and $T_{2}$, and two control parameters, TE and TR. We model images as Markov random fields and introduce four maximum a posteriori (MAP) restorations, nonlinear techniques using several different prior terms which reduce the correlated noise in the basis images, thereby reducing the noise in the restored MR images. The "product" and "sum" forms for basis (signal) and spatial correlations are discussed, compared and evaluated for various situations and features.

\section{INTRODUCTION}

Magnetic Resonance Imaging (MRI) has proven to be a very useful noninvasive medical imaging method because of the ability to render high anatomical resolution of soft tissues.

Three variables form a basis for MR images: proton density, and two nuclear relaxation times. MRI systems can produce multiple images which emphasize one or several of these three basis variables while enforcing a reasonable level of registration between images, by adjusting one or more of the user-specified parameters, TE and TR.

At the present time, MR images are often processed or viewed individually, so some important clinical details may go unnoticed due to not using the joint information between multiple images. This problem can be avoided if an approach which incorporates multiple images is utilized, and done in a manner such that important basis images are extracted. Once this is done, synthetic images may be calculated from the basis images. These synthetic images can be used to generate images equivalent to data images obtainable with different control parameters, TE and TR, thereby minimizing the MR system time [17] [21] [6] [8]. 
In this work, the unknown basis images and the MR images are modeled as Markov random fields. The basis images are related to the MR images through a physical model. Four MAP restorations are compared; nonlinear priors that are biased towards piecewise-smooth basis images and which reduce the correlated noise in the basis images. The "product" (AND) and "sum" (OR) forms [3] for basis (signal) and spatial correlations are discussed. The application of non-quadratic prior probabilities requires global optimization. Here we present results of the nonlinear prior work, along with error landscapes generated during restorations of synthetic data. Our choice of nonlinear prior is based on our previous experience [2] with several approaches towards the nonlinear restoration of images. MR images synthesized by using the restored basis images are compared to the original MR data.

Section 2 briefly discusses the results of our previous work [8] and other research. Section 3 presents useful information necessary for understanding our approach. In Section 4, the new contributions of signal and spatial "AND" and "OR" prior terms are discussed, and Section 5 reports experimental results.

\section{BACKGROUND}

The purpose of this section is to briefly discuss previous work in the determination of the MRI basis images (proton density and $T_{1}$ and $T_{2}$ nuclear relaxation times), and the restoration of noisy MRI data using these basis images.

In MRI, typical images are proton density weighted images, $T_{1}$ weighted images, and $T_{2}$ weighted images, which are acquired through selection of appropriate values of TE (echo time) and TR (relaxation time). At the present time, abnormal tissue areas are examined by the contrast in each image, and these images are viewed and treated separately by radiologists. Because of this single-image approach, MRI pulse sequences were developed to enhance the contrast of the characteristics of a particular tissue. However, through the utilization of routinely used "multi-echo" pulse sequences, several pixel-registered images can be obtained which reflect the different tissue properties. By analyzing these multiple pixel-registered images, the proton density, and the $T_{1}$ and $T_{2}$ nuclear relaxation times may be obtained for each sampled point in the multiple-image set.

Graumann, Fischer and Oppelt [12] designed a specific pulse sequence in order to directly calculate $T_{1}$ and $T_{2}$ without the use of minimization techniques. Brosnan, et al. [7] discuss an improvement over Wiener filtering, "measurement-dependent filtering", which uses multiple data sources to decrease the noise in a previously calculated $T_{2}$ image while maintaining step edges. Bobman, et al. [5] [4] and Wright, et al. [22] did not apply noise statistics while performing linear regression. MacFall, Riederer and Wang [19] and Liu, Nieminen and Koenig [18] used $\chi^{2}$ techniques on log-transformed data, and used a log-transformed variance during minimization. While these methods [19] [18] deal with noise distributions more appropriately than those previously described [5] [4] [22], log-transformations exhibit reduced performance in low SNR cases, since the transformed noise distribution is distorted from the Gaussian model, yet the $\chi^{2}$ model assumes a truly Gaussian distribution. Furthermore, the cited papers [19] [18] [5] [4] [22], do not present algorithms which simultaneously determine proton density, $T_{1}$ and $T_{2}$. Instead they approximate a nonlinear multivariate (3-D) problem into a set of linear 1-D or 2-D problems by a variety of nonlinear transformations on the system of equations, thereby distorting the noise distributions and propagating error into another basis image. They also cannot determine proton density, $T_{2}$ and $T_{1}$ basis signals 
unless they have acquired two or more images with the same TE, and two or more images with the same TR. Our approach does not have these restrictions. The $\chi^{2}$ approach is a special case of the maximum likelihood (ML) (minimum variance least-squares) approach, and is expected to produce results no better than our ML restorations. $N$ data images may be used to extract proton density, $T_{1}$ and $T_{2}$ in the ML algorithm. If there are $M$ pixels within a single contiguous region of the proton density, $T_{1}$ and $T_{2}$ images possessing similar statistics, then the MAP algorithm uses on the order of $N \times M$ data pixels to develop the restoration value of a single pixel of interest in that region. Approximately $N \times M$ data images would be necessary to produce an ML result with equivalent noise reduction to the MAP result for that single pixel of interest.

In all of the cited cases, the basis images have been calculated without the benefit of any $a$ priori knowledge of the local characteristics of the tissues of interest. To date, with the exception of our previous work [8], only data images have been processed with the benefit of prior knowledge [11] [14] [10] [13]. It has been our intent to use just such a priori knowledge to reduce the noise and increase the contrast within the basis images and subsequently synthesized restorations, while dealing with noisy data more explicitly than previously presented.

\section{PROBLEM FORMULATION}

The purpose of this section is to develop the definitions and mathematical basis for further discussion of our approach.

Let $\mathbf{G}$ be a measured vector-set of images

$$
\begin{aligned}
& \mathrm{G}=\left\{\mathbf{g}_{c}\right\}_{c=1, d} \\
& \mathbf{g}_{c}=\left[g_{c, i}\right]_{i=1, n}
\end{aligned}
$$

where $d$ is the number of channels in the vector-set, and where $g_{c, i}$ represents the $c$-th channel value associated with the $i$-th pixel.

Using similar notation, let $\mathbf{S}(\mathbf{F})$ represent the undegraded ideal images as a deterministic function of $\mathbf{F}$ where $\mathbf{F}$ are the undegraded ideal basis images, and let $\mathrm{N}$ represent additive noise such that $\mathbf{G}=\mathbf{S}+\mathbf{N}$. Note that

$$
\begin{aligned}
& \mathbf{F}=\left\{\mathbf{f}_{\psi}\right\}_{\psi=1, p} \\
& \mathbf{f}_{\psi}=\left[f_{\psi, i}\right]_{i=1, n}
\end{aligned}
$$

where $p$ is the number of basis images in the vector-set, and where $f_{\psi, i}$ represents the value associated with the $i$-th pixel of the $\psi$-th basis image.

\subsection{Bayesian Model}

In Bayesian restoration, the most acceptable result is the result with the highest probability of occurrence. Let $\hat{\mathbf{F}}$ be an estimate of $\mathbf{F}$. Bayes' rule gives the posterior distribution [9] of $\hat{\mathbf{F}}$ given the data $\mathbf{G}$ as

$$
P(\hat{\mathbf{F}} \mid \mathbf{G})=\frac{\mathrm{P}(\mathbf{G} \mid \hat{\mathbf{F}}) \mathrm{P}(\hat{\mathbf{F}})}{\mathrm{P}(\mathbf{G})}
$$

That is, the conditional probability of occurrence of a specific restoration $\hat{\mathbf{F}}$ given the data $\mathbf{G}$ is equal to the conditional probability of occurrence of the data $G$ given the specific restoration $\hat{F}$ 
times the probability of the occurrence of the specific restoration $\hat{\mathbf{F}}$ divided by the probability of the occurrence of the data $\mathbf{G}$. We refer to $P(\mathbf{G} \mid \hat{\mathbf{F}})$ as the "noise term", and it describes the noise distribution. $\mathrm{P}(\hat{\mathbf{F}})$ is called the "prior term" and it describes the a priori distribution which can be chosen using a priori knowledge about $\mathbf{F}$. Obviously $\mathrm{P}(\mathbf{G})$ is constant and independent of $\hat{\mathbf{F}}$, so in order to maximize the posterior distribution, we need only maximize $P(\mathbf{G} \mid \hat{\mathbf{F}}) \mathrm{P}(\hat{\mathbf{F}})$.

The determination of the cost function, and the noise and prior terms of that cost function are presented in Section 4.

\subsection{Physical Model}

The function $\mathbf{S}(\mathbf{F})$ is given by the physical model. In this work, one simplified nonlinear image formation model [18] is used.

$$
s_{c, i}=\rho_{i} \exp \left(-\mathrm{TE}_{c} / T_{2 i}\right)\left\{1-\exp \left(-\mathrm{TR}_{c} / T_{1 i}\right)\right\}
$$

where $\rho, \mathrm{T}_{2}$ and $\mathrm{T}_{1}$ are basis images of $\mathbf{f}_{\psi}$ where $\psi=1,2,3$, respectively. $\mathrm{TE}_{c}$ and $\mathrm{TR}_{c}$ represent the echo time and relaxation time used during acquisition of the $c$-th data image. $T_{1}$ and $T_{2}$ are nuclear relaxation times, and $\rho$ represents proton density, contributions due to proton flow, and MRI system gain. Most brain tissue is perfuse with slowly moving blood, hence the data should not be subject to large variations in proton flow. This work does not address the effect of proton flow. Our data was acquired with MRI system gain held constant for all scans.

Note that this physical model is undefined and exhibits singularities in the gradient when $T_{1}$ or $T_{2}$ equals zero. $T_{1}$ and $T_{2}$ are real, positive and bounded below in time, but using a noninfinitesimal step size during gradient descent requires that $T_{1}$ and $T_{2}$ be constrained in code, otherwise negative values of $T_{1}$ or $T_{2}$ might occur, causing numerical overflow. Because of this, a constrained optimization technique is required to find a global solution in the minimization process.

\section{IMAGE RESTORATION}

This section delineates the different approaches we have taken towards restoration of the basis images through deterministic minimization of a cost function. In all cases, a maximum likelihood (ML) ("noise" ) term is necessary to ensure fidelity to the data. A "prior" term is introduced into the minimization scheme only when neighborhood interactions are necessary to correct the effects of noise-corrupted data. This noise propagates in a nonlinear and signal-dependent manner [19] [1] into the basis images described within the physical model. The "prior" term addresses this effect by operating on the basis images within a local neighborhood.

\subsection{The Noise Term, $H_{N}$}

The ML approach does not incorporate a prior model, hence the restoration is based solely on a noise term. For the ML case, the noise which has corrupted $\mathbf{S}(\mathbf{F})$ is modeled as stationary, signal-independent additive Gaussian noise $[19]$, hence $P(\mathbf{G} \mid \hat{\mathrm{F}})$ is given by

$$
\mathrm{P}(\mathbf{G} \mid \hat{\mathbf{F}})=\mathrm{P}(\mathbf{N}) \equiv \frac{\exp \left(-\frac{1}{2} \mathbf{N}^{\mathrm{T}} \mathbf{C}^{-1} \mathbf{N}\right)}{(2 \pi)^{n d / 2}|\mathbf{C}|^{1 / 2}}
$$

where $\mathbf{C}$ denotes the covariance matrix of noise $\mathbf{N}$, and $|\mathbf{C}|$ denotes the determinant of $\mathbf{C}$. Since the noise is assumed to be zero-mean, $\mathbf{C}=\mathbf{R}$, the autocorrelation matrix, and Equation 5 can now 
be written as

$$
\begin{gathered}
\mathrm{P}(\mathbf{G} \mid \hat{\mathbf{F}})=\frac{\exp \left(-\frac{1}{2}(\hat{\mathbf{S}}(\hat{\mathbf{F}})-\mathbf{G})^{\mathrm{T}} \mathbf{R}^{-1}(\hat{\mathbf{S}}(\hat{\mathbf{F}})-\mathbf{G})\right)}{(2 \pi)^{n d / 2}|\mathbf{R}|^{1 / 2}}= \\
\exp \left(K-\frac{1}{2}(\hat{\mathbf{S}}(\hat{\mathbf{F}})-\mathbf{G})^{\mathrm{T}} \mathbf{R}^{-1}(\hat{\mathbf{S}}(\hat{\mathbf{F}})-\mathbf{G})\right)
\end{gathered}
$$

where $K$ is some constant value. Taking the negative-log of $\mathrm{P}(\mathrm{G} \mid \hat{\mathrm{F}})$, and harmlessly ignoring the constant contribution of $K$, the "noise term" of the optimization function is obtained:

$$
\begin{gathered}
H_{N}(\hat{\mathbf{F}}, \mathbf{G})=\frac{1}{2}(\hat{\mathbf{S}}(\hat{\mathbf{F}})-\mathbf{G})^{\mathrm{T}} \mathbf{R}^{-1}(\hat{\mathbf{S}}(\hat{\mathbf{F}})-\mathbf{G})= \\
\sum_{c} \frac{1}{2 \sigma_{c}{ }^{2}} \sum_{i}\left(\hat{s}_{c, i}(\hat{\mathbf{F}})-g_{c, i}\right)^{2}
\end{gathered}
$$

for isotropic uncorrelated noise.

In order to ascertain the ML estimates of $\rho, T_{2}$ and $T_{1}$ images, we arbitrarily set the initial basis images to the following values:

$$
\begin{gathered}
T_{1 i}=\frac{1}{N} \sum_{c=0}^{N-1} \mathrm{TR}_{c} \\
T_{2 i}=\frac{1}{N} \sum_{c=0}^{N-1} \mathrm{TE}_{c} \\
\rho_{i}=\frac{1}{N} \sum_{c=0}^{N-1} g_{c, i} \exp \left(\mathrm{TE}_{c} / T_{2 i}\right)\left\{1-\exp \left(-\mathrm{TR}_{c} / T_{1 i}\right)\right\}^{-1}
\end{gathered}
$$

For the case where only ML restoration is attempted, the cost function is $H=H_{N}$.

\subsection{The Prior Term, $H_{P}$}

Smoothing requires the use of an additional term in the cost function, the prior term. This term, $\mathrm{P}(\hat{\mathbf{F}})$, depends only on $\hat{\mathbf{F}}$, and should reflect some prior knowledge of the nature of $\mathbf{F}$. For the case of MR images, the basis images should appear to be locally homogeneous in some important way. $\mathrm{P}(\hat{\mathbf{F}})$ :

Since it is frequently used in similar restoration problems [9], an exponential form is chosen for

$$
\mathrm{P}(\hat{\mathbf{F}})=\frac{\exp \left(-H_{p}(\hat{\mathbf{F}})\right)}{Z_{p}}
$$

where $Z_{p}$ normalizes $\mathrm{P}(\hat{\mathbf{F}}) . H_{p}(\hat{\mathrm{F}})$ in this instance is used to measure and regulate the local homogeneity of some characteristic of the basis images.

$\mathrm{P}(\hat{\mathbf{F}})$ can take on many different forms. Approaches include the "product" (AND) and "sum" (OR) forms [3], since the probability may be expressed as a product or sum of exponentials, respectively. We shall hereinafter refer to these forms as AND and OR forms, since the neighborhood interactions of $H_{p}\left(\hat{\mathbf{F}}_{i}\right)$ behave in the logical AND and OR manner. That is, in using the AND form for nonlinear spatial priors, any single neighborhood interaction may saturate $H_{p}\left(\hat{\mathrm{F}}_{i}\right)$ despite the interactions of other clique members, resulting in a restoration which may have noisy edges. With the OR form, a single neighborhood interaction cannot zero the prior gradient contribution. We 
extend the use of this terminology from the spatial correlation case to the basis (signal) correlation case, since we will use this approach to deal with multiple signals correlated through a physical model.

It is obvious that by reducing the noise in the basis images, we reduce the noise in the restored data. If we use the basis covariance matrix in the noise reduction process, we are assured that the correlation matrices of both the restored data and restored basis image are changed only a small amount from the ML case. This can be seen by assuming a piecewise constant region over $F$ and therefore $\mathbf{S}(\mathbf{F})$, and noting that

$$
H_{N}(\hat{\mathbf{F}}, \mathbf{G})=\frac{1}{2} \sum_{i}\left(\mathbf{S}\left(\hat{\mathbf{F}}_{i}\right)-\mathbf{G}_{i}\right)^{\mathrm{T}} \mathbf{C}_{\mathbf{G}}^{-1}\left(\mathbf{S}\left(\hat{\mathbf{F}}_{i}\right)-\mathbf{G}_{i}\right)
$$

Given that

$$
\mathbf{C}_{\mathbf{G}}=\left\langle\left(\mathbf{S}\left(\mathbf{F}_{i}\right)-\mathbf{G}_{i}\right)\left(\mathbf{S}\left(\mathbf{F}_{i}\right)-\mathbf{G}_{i}\right)^{\mathrm{T}}\right\rangle-\left\langle\left(\mathbf{S}\left(\mathbf{F}_{i}\right)-\mathbf{G}_{i}\right)\right\rangle\left\langle\left(\mathbf{S}\left(\mathbf{F}_{i}\right)-\mathbf{G}_{i}\right)^{\mathrm{T}}\right\rangle
$$

we approximate

$$
\left.\mathbf{S}\left(\mathbf{F}_{i}\right) \approx \mathbf{S}\left(\mathbf{F}_{i}\right)\right|_{\mathbf{F}_{i}=\hat{\mathbf{F}}_{i}}+\left.\frac{\partial \mathbf{S}\left(\mathbf{F}_{i}\right)}{\partial \mathbf{F}_{i}}\right|_{\mathbf{F}_{i}=\hat{\mathbf{F}}_{i}}\left(\mathbf{F}_{i}-\hat{\mathbf{F}}_{i}\right)
$$

where $\mathbf{F}_{i}=\hat{\mathrm{F}}_{i}$ is the ML solution which minimizes Equation 12 for the $i$-th pixel-vector. Substituting this relation into Equation 13, we find

$$
\begin{aligned}
& \mathbf{C}_{\mathbf{G}} \approx\left\langle\left(\mathbf{S}\left(\hat{\mathbf{F}}_{i}\right)+\frac{\partial \mathbf{S}\left(\hat{\mathbf{F}}_{i}\right)}{\partial \mathbf{F}_{i}}\left(\mathbf{F}_{i}-\hat{\mathbf{F}}_{i}\right)-\mathbf{G}_{i}\right)\left(\mathbf{S}\left(\hat{\mathbf{F}}_{i}\right)+\frac{\partial \mathbf{S}\left(\hat{\mathbf{F}}_{i}\right)}{\partial \mathbf{F}_{i}}\left(\mathbf{F}_{i}-\hat{\mathbf{F}}_{i}\right)-\mathbf{G}_{i}\right)^{\mathrm{T}}\right\rangle \\
&-\left\langle\left(\mathbf{S}\left(\hat{\mathbf{F}}_{i}\right)+\frac{\partial \mathbf{S}\left(\hat{\mathbf{F}}_{i}\right)}{\partial \mathbf{F}_{i}}\left(\mathbf{F}_{i}-\hat{\mathbf{F}}_{i}\right)-\mathbf{G}_{i}\right)\right\rangle\left\langle\left(\mathbf{S}\left(\hat{\mathbf{F}}_{i}\right)+\frac{\partial \mathbf{S}\left(\hat{\mathbf{F}}_{i}\right)}{\partial \mathbf{F}_{i}}\left(\mathbf{F}_{i}-\hat{\mathbf{F}}_{i}\right)-\mathbf{G}_{i}\right)^{\mathrm{T}}\right\rangle \\
& \mathbf{C}_{\mathbf{G}} \approx\left\langle\frac{\partial \mathbf{S}\left(\hat{\mathbf{F}}_{i}\right)}{\partial \mathbf{F}_{i}}\left(\mathbf{F}_{i}-\hat{\mathbf{F}}_{i}\right)\left(\mathbf{F}_{i}-\hat{\mathbf{F}}_{i}\right)^{\mathrm{T}} \frac{\partial \mathbf{S}\left(\hat{\mathbf{F}}_{i}\right)}{\partial \mathbf{F}_{i}}\right\rangle-\left\langle\frac{\partial \mathbf{S}\left(\hat{\mathbf{F}}_{i}\right)}{\partial \mathbf{F}_{i}}\left(\mathbf{F}_{i}-\hat{\mathbf{F}}_{i}\right)\right\rangle\left\langle\left(\mathbf{F}_{i}-\hat{\mathbf{F}}_{i}\right)^{\mathrm{T}} \frac{\partial \mathbf{S}\left(\hat{\mathbf{F}}_{i}\right)^{\mathrm{T}}}{\partial \mathbf{F}_{i}}\right\rangle
\end{aligned}
$$

We make the additional approximation that over a piecewise-constant region, $\partial \mathbf{S}\left(\hat{\mathbf{F}}_{i}\right) / \partial \mathbf{F}_{i}$ may be moved outside the expectation operator. Hence,

$$
\mathbf{C}_{\mathbf{G}} \approx\left\langle\frac{\partial \mathbf{S}\left(\hat{\mathbf{F}}_{i}\right)}{\partial \mathbf{F}_{i}}\right\rangle \mathbf{C}_{\mathbf{F}}\left\langle\frac{\partial \mathbf{S}\left(\hat{\mathbf{F}}_{i}\right)^{\mathrm{T}}}{\partial \mathbf{F}_{i}}\right\rangle
$$

In this work, we investigate four nonlinear forms of $\mathrm{P}(\hat{\mathbf{F}})$. These four nonlinear forms have two corresponding linear forms which are presented in Section 4.2.1. The first nonlinear form uses a signal-OR, spatial-AND approach, and is discussed in Section 4.2.2. The second form utilizes a signal-OR, spatial-OR approach, and is discussed in Section 4.2.3. The third form applies a signal-AND, spatial-AND approach, and is discussed in Section 4.2.4. The fourth form uses a signal-AND, spatial-OR approach, and is discussed in Section 4.2.5.

4.2.1 The Quadratic Prior Terms, $H_{P}$

For the case of signal-OR quadratic smoothing, we choose

$$
H_{p}(\hat{\mathbf{F}})=\sum_{\psi} \frac{\lambda}{2 \sigma_{\psi}^{2}} \sum_{i} \sum_{\eta} w_{\eta}\left(\frac{\partial \hat{f}_{\psi, i}}{\partial x_{\eta}}\right)^{2}
$$


where $\eta$ indicates the direction of the first-order partial differential, $w_{\eta}$ adjusts the relative contribution of nearest-neighbor versus next-nearest-neighbor pixels, and $\lambda$, in conjunction with the estimate of noise in the $\psi$-th image, $\sigma_{\psi}$, indicates the emphasis of smoothing on the basis images.

For the case of signal-AND quadratic smoothing, we choose

$$
H_{p}(\hat{\mathbf{F}})=\frac{\lambda}{2} \sum_{i} \sum_{\eta} w_{\eta}\left(\frac{\partial \hat{\mathbf{F}}_{i}}{\partial x_{\eta}}\right)^{\mathrm{T}} \mathbf{C}_{\mathbf{F}}^{-1}\left(\frac{\partial \hat{\mathbf{F}}_{i}}{\partial x_{\eta}}\right)
$$

where $\mathbf{C}_{\mathbf{F}}{ }^{-1}$ is the estimated covariance of the basis images.

To determine the smoothed values for the $\rho, T_{2}$ and $T_{1}$ images, we initialize these values with the method put forth in Equations 8, 9 and 10. When neighborhood interactions are used, the cost function is written as $H=H_{N}+H_{P}$.

Quadratic smoothing performs well in regions where the basis images do not exhibit large excursions or contain step edges. In areas where the ML solution yields such large excursions, quadratic smoothing unacceptably blurs these features. The advantage of nonlinear smoothing is that it can preserve these features while maintaining the same level of smoothing performance on those regions where large excursions do not exist.

\subsubsection{The Nonlinear Signal-OR, Spatial-AND Prior Term}

For the case where nonlinear piecewise smoothing is desirable, a previously reported [8] prior with known advantages is

$$
H_{p}(\hat{\mathbf{F}})=\sum_{\psi} \sum_{i} \frac{\lambda \sum_{\eta} w_{\eta}\left(\frac{\partial f_{\psi, i}}{\partial x_{\eta}}\right)^{2}}{2 \sigma_{\psi}^{2}+\frac{\lambda}{\tau_{\psi}^{2}} \sum_{\eta} w_{\eta}\left(\frac{\partial f_{\psi, i}}{\partial x_{\eta}}\right)^{2}}
$$

where $\lambda$ is a constant and $\tau_{\psi}$ is a smoothly changing annealing parameter on the $\psi$-th basis image. At infinite $\tau_{\psi}$ for all $\psi$, the prior term is equivalent to the previously discussed quadratic prior term put forth in Equation 14. As $\tau_{\psi}$ is decreased, the influence of the prior term gives way to the noise term, and the restoration takes on the appearance of the ML solution as $\tau_{\psi} \rightarrow 0$. For the ML case, the solution for reasonable SNR is obtained within a system with a single local minimum. For the quadratic smoothing case, the prior term is convex. Figure 1 illustrates the nonlinear prior function at several temperatures for the function $v(x)=x^{2} /\left(1+\frac{x^{2}}{\tau^{2}}\right)$.

For the case of nonlinear restoration, we initialize the basis images with the output from the quadratic smoothing case. We require the annealing schedule have an initial $\tau_{\psi}$ large enough that the prior energy cost function is convex for the entire $\psi$-th basis image. The interim restoration for the initial $\tau_{\psi}$ should appear to approximate the final restoration for quadratic smoothing. We arbitrarily set the initial $\tau_{\psi}=10 \forall \psi$. Once the minimum has been obtained for that initial temperature, $\tau_{\psi}$ is reduced by a constant multiplier, $k=0.9$, and a minimum is again sought. This continues until an acceptable restoration is obtained. For the nonlinear prior of Equation 16 the final $\tau_{\psi}$ has been empirically determined to be approximately 1 for all $\psi$ when applied to clinical data. In general, the number of minimizations, $N$, is determined by

$$
N=\frac{\log \tau_{\psi, f \text { inal }}-\log \tau_{\psi, \text { init }}}{\log k} .
$$




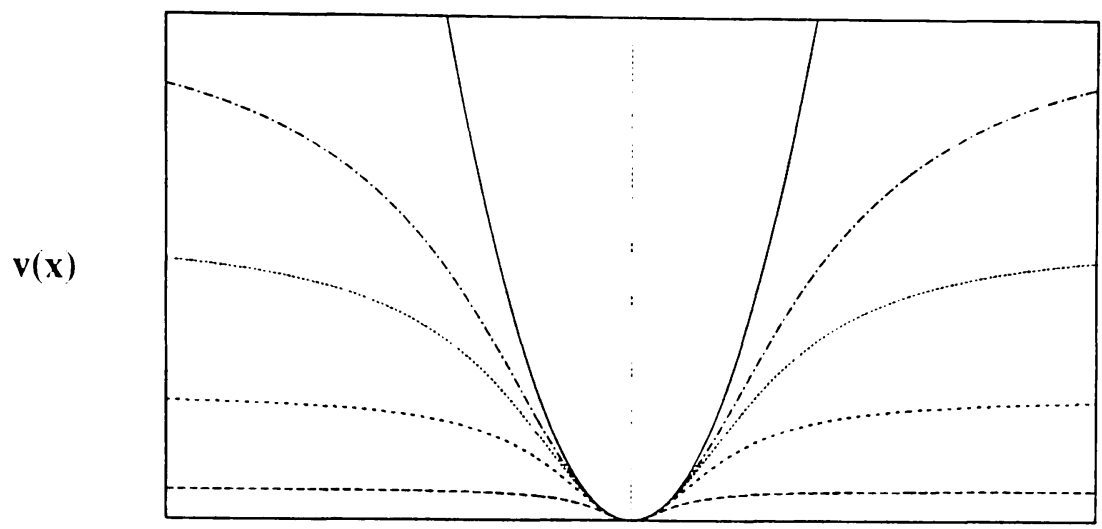

$\mathbf{x}$

Figure 1: The nonlinear prior function at several temperatures.

By choosing a reasonable annealing schedule, these final $\tau$ values produce a piecewise smooth restoration between the quadratically smoothed and ML solutions; that is, a restoration that is both locally smooth and yet retains fidelity to the data by preserving step edges. In this way, we start from an overly smoothed restoration, and anneal to a final restoration which does not overly suppress single-pixel disturbances which a radiologist might find important.

\subsubsection{The Nonlinear Signal-OR, Spatial-OR Prior Term}

In the spatial-AND approach in nonlinear restoration, any single contributing clique-member interacting with a pixel-of-interest can saturate the prior for all other clique-members; the prior gradient effectively vanishes, and the pixel-of-interest reverts to the ML solution. This is often the case at step edges. By modifying the prior to take a spatial-OR approach, a clique member can only saturate its own contribution, and so other clique members are free to contribute to the local estimate of the pixel-of-interest. Another nonlinear piecewise smooth prior we use is

$$
H_{p}(\hat{\mathbf{F}})=\sum_{\psi} \sum_{i} \sum_{\eta} \frac{\lambda w_{\eta}\left(\frac{\partial f_{\psi, i}}{\partial x_{\eta}}\right)^{2}}{2 \sigma_{\psi}{ }^{2}+\frac{\lambda}{\tau_{\psi}} w_{\eta}\left(\frac{\partial f_{\psi, i}}{\partial x_{\eta}}\right)^{2}} .
$$

At infinite $\tau_{\psi}$ for all $\psi$, the prior term is equivalent to the previously discussed quadratic prior term put forth in Equation 14. For the nonlinear prior of Equation 18 the final $\tau_{\psi}$ has been empirically determined to be approximately 0.25 for all $\psi$ when applied to clinical data.

\subsubsection{The Nonlinear Signal-AND, Spatial-AND Prior Term}

Due to the nonlinear physical model, the independent noise in the data images has propagated as highly correlated noise into the basis images. We use a covariance matrix to incorporate some 
measure of the correlated noise distribution within the basis images.

$$
H_{p}(\hat{\mathbf{F}})=\sum_{i} \frac{\frac{\lambda}{2} \sum_{\eta} w_{\eta}\left(\frac{\partial \hat{\mathbf{F}}_{i}}{\partial x_{\eta}}\right)^{\mathrm{T}} \mathbf{C}_{\mathbf{F}}^{-1}\left(\frac{\partial \hat{\mathbf{F}}_{i}}{\partial x_{\eta}}\right)}{1+\frac{\lambda}{2 \tau^{2}} \sum_{\eta} w_{\eta}\left(\frac{\partial \hat{\mathbf{F}}_{i}}{\partial x_{\eta}}\right)^{\mathrm{T}} \mathbf{C}_{\mathbf{F}}^{-1}\left(\frac{\partial \hat{\mathbf{F}}_{i}}{\partial x_{\eta}}\right)}
$$

At infinite $\tau$, the prior term is equivalent to the previously discussed quadratic prior term put forth in Equation 15. For the nonlinear prior of Equation 19 the final $\tau$ has been empirically determined to be approximately 1 when applied to clinical data.

\subsubsection{The Nonlinear Signal-AND, Spatial-OR Prior Term}

The nonlinear prior term for the signal-AND, spatial-OR case is

$$
H_{p}(\hat{\mathbf{F}})=\sum_{i} \sum_{\eta} \frac{\frac{\lambda w_{\eta}}{2}\left(\frac{\partial \hat{\mathbf{F}}_{i}}{\partial \boldsymbol{x}_{\eta}}\right)^{\mathrm{T}} \mathbf{C}_{\mathbf{F}}^{-1}\left(\frac{\partial \hat{\mathbf{F}}_{i}}{\partial \boldsymbol{x}_{\eta}}\right)}{1+\frac{\lambda w_{\eta}}{2}\left(\frac{\partial \hat{\mathbf{F}}_{i}}{\partial \boldsymbol{x}_{\eta}}\right)^{\mathrm{T}} \mathbf{C}_{\mathbf{F}}{ }^{-1}\left(\frac{\partial \hat{\mathbf{F}}_{i}}{\partial \boldsymbol{x}_{\eta}}\right)} .
$$

The prior term is equivalent to the previously discussed quadratic prior term put forth in Equation 15 at infinite $\tau$. The final $\tau$ has been empirically determined to be approximately 0.25 for the nonlinear prior of Equation 20 when applied to clinical data.

\section{EXPERIMENTAL RESULTS}

This section is divided into three major subsections which separately discuss restoration results on synthetic, phantom and clinical data. The synthetic data was used to test restoration quality with the application of an objective measurement of error. The phantom data was used to test restoration quality against simple-averaged multiply-acquired data sets. The real MRI data was used to study the clinical usefulness of the four nonlinear priors applied into our restoration algorithm.

\subsection{Synthetic Data}

Synthetic basis images were designed using the mean values of tissues (white matter, gray matter, sulci, and two different lesions) obtained from the ML basis image restorations of a human brain exhibiting some pathological disturbances. Noiseless MR data images were generated from these synthesized basis images using the physical model, and are shown in the lower-left corner of Figures 6 through 11. The noisy synthetic data were then obtained by adding zero-mean Gaussian noise to the noiseless MR images. The statistics for the additive noise were obtained from the real MR data images of the previously mentioned human brain. (See Figures 15, 16 and 17.) All TE and TR values used in this synthesis were identical to those used in the acquisition of the real brain data.

The noisless images in the lower-left of Figures 6 through 11 contain four features and background with values closely approximating the mean of certain areas of the clinical data in Figures 15, 16 and 17. The "table" in the upper-left region of the synthetic images corresponds to the large, diffuse lesion in the lower-left regions of Figures 15, 16 and 17. The square "ring" and its interior 
pixels in the lower-left region and the "spike" (single-pixel feature) in the lower-center region of the synthetic images correspond to the ring and interior formed by the cyst-like lesion in the upper region of Figures 15, 16 and 17. The "dog-leg" in the right region of the synthetic images and the background correspond to the sulci and white-matter, respectively, in Figures 15, 16 and 17.

Figures 2, 3, 4 and 5 illustrate the RMS error for the data restorations using the nonlinear priors presented in Section 4.2, utilizing only the diagonal elements of the white matter covariance. It is unnecessary and perhaps too restrictive to implement the full covariance matrix, since the interaction of the noise term through the physical model ensures reasonable fidelity to the full covariance, and because the off-diagonal elements can change dramatically between piecewise smooth regions of differing tissues. These error surfaces are parameterized by $\lambda$, the smoothing factor, and $\tau$, the annealing temperature. Note that the constant error plateau at low temperature is due to the restoration reverting to the ML solution. Also note that the error surfaces for the spatial-AND priors have a rougher terrain than those of the spatial-OR priors due to the saturation effect of a single-neighbor interaction; the rough terrain on the low-temperature side of the minimum error trough is caused by single pixels at a step edge reverting to the ML solution at slightly different $\tau$ and $\lambda$.

\section{Restoration RMS Error: Signal-OR, Spatial-AND}

\section{minimum RMS error 15.83}

\section{maximum RMS error 71.3}

error

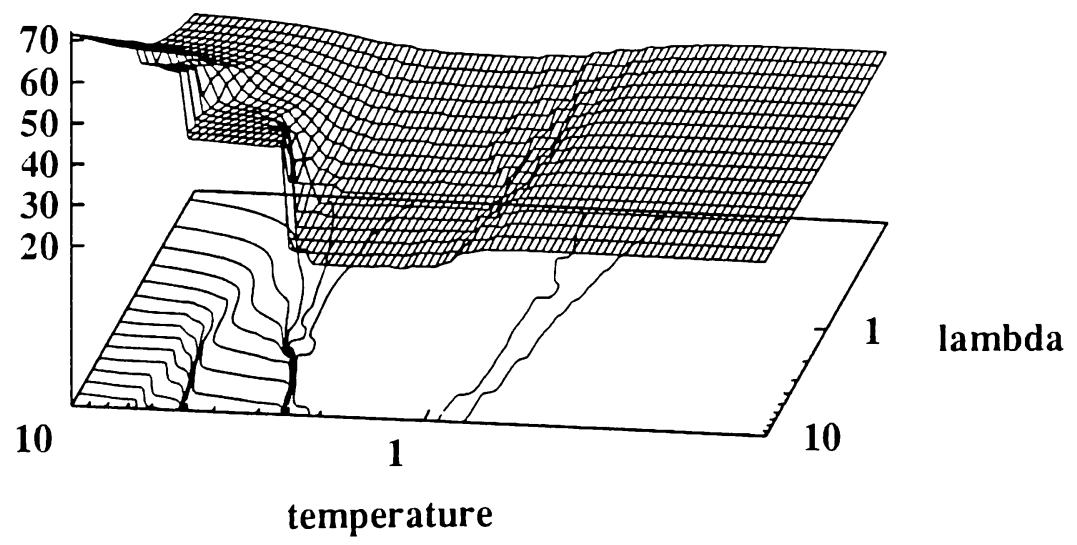

Figure 2: RMS Error Surface for Signal-OR, Spatial-AND Prior

The synthetic data and best restorations using $\lambda=1$ are presented in Figures 6 through 8 which represent data and restorations with $(\mathrm{TE}, \mathrm{TR})=(32,3000),(90,3000)$, and $(17,417)$, respectively. The images in the top row are, left-to-right, the signal-OR, spatial-AND restoration, and the signal-OR, spatial-OR restoration, The middle row is composed of the signal-AND, spatial-AND restoration on the left, and the signal-AND, spatial-OR restoration. Left-to-right, the bottom row images are the noise-free data and the data with noise. All images within each figure are videoscaled 
The Effect of Various Basis Image Priors on MR Image MAP Restoration

Restoration RMS Error: Signal-OR, Spatial-OR

minimum RMS error 10.75

maximum RMS error 71.6

error

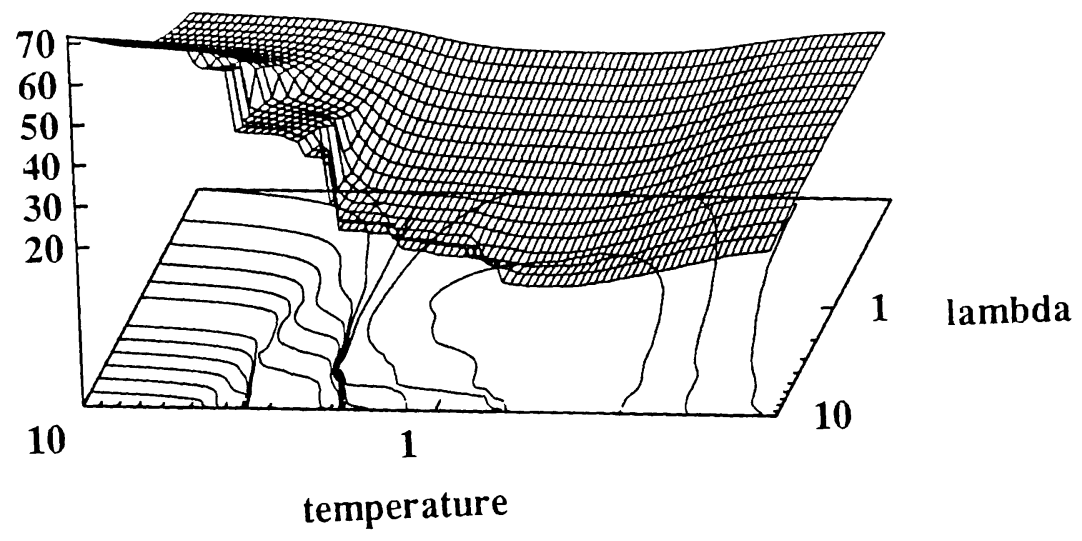

Figure 3: Error Surface for Signal-OR, Spatial-OR Prior

Restoration RMS Error: Signal-AND, Spatial-AND

minimum RMS error 15.56

maximum RMS error 71.8

error

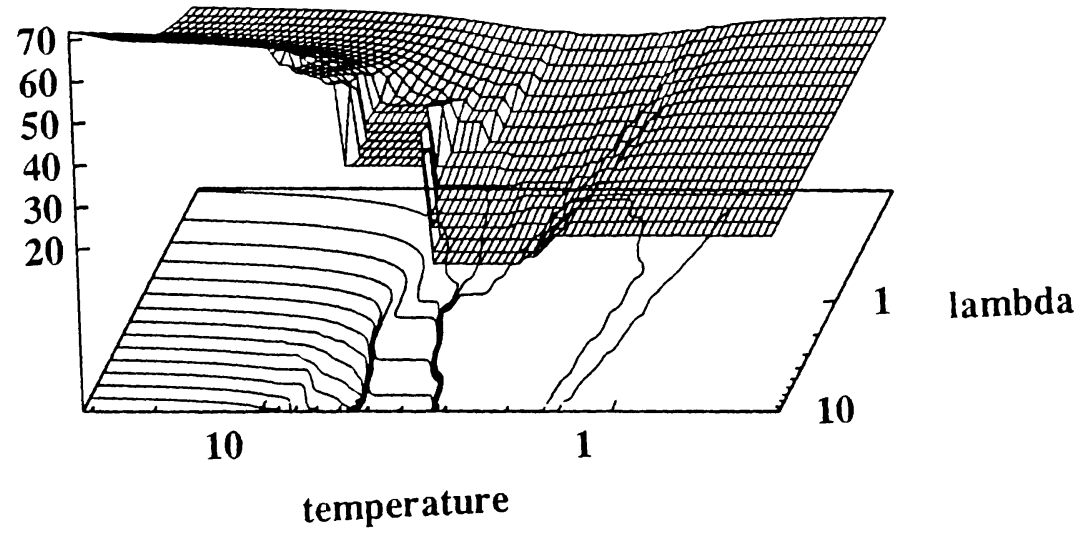

Figure 4: Error Surface for Signal-AND, Spatial-AND Prior 


\section{Restoration RMS Error: Signal-AND, Spatial-OR}

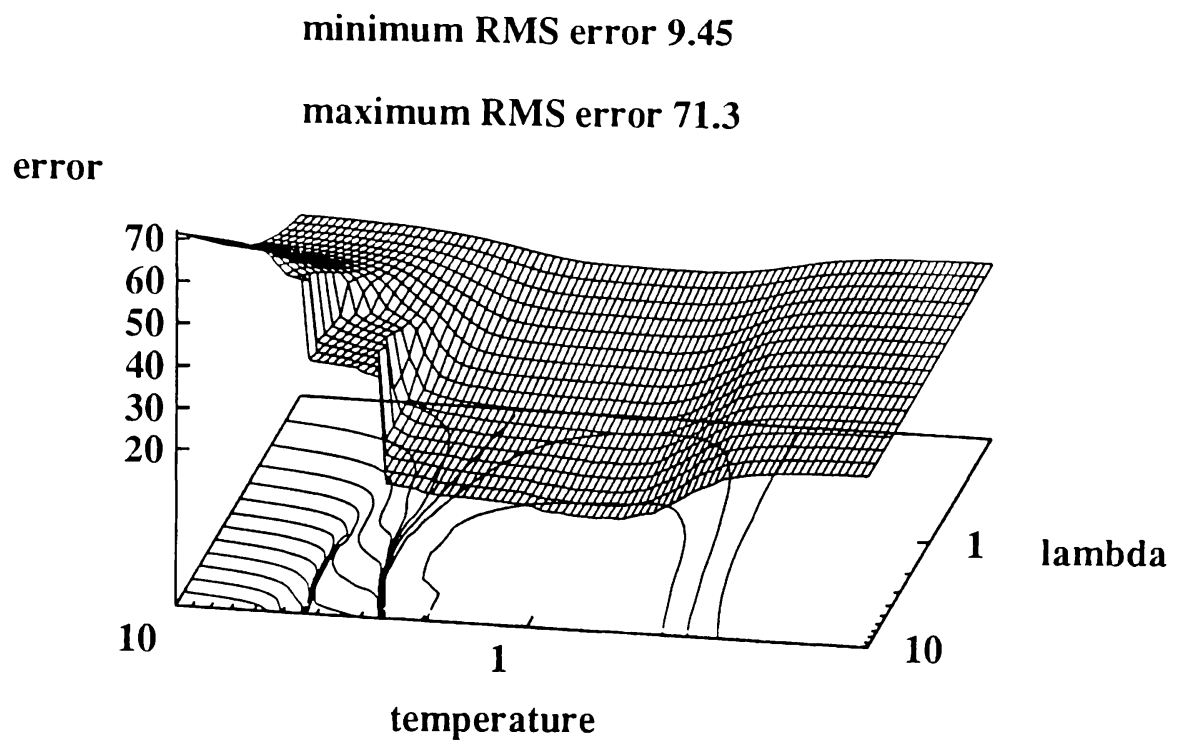

Figure 5: Error Surface for Signal-AND, Spatial-OR Prior

to a common scale. The spatial-OR priors preserve step edges better than the spatial-AND priors, as evidenced by observing the ring, table and dog-leg in Figures 6 through 8 . It can be observed that the signal-AND, spatial-OR restoration is superior to the signal-OR, spatial-OR restoration by examining the dog-leg in the upper-right and center-right frames of Figure 8 .

The synthetic data and best restorations as determined by minimum RMS error are presented within Figures 9 through 11 which represent data acquired with $(\mathrm{TE}, \mathrm{TR})=(32,3000),(90,3000)$, and $(17,417)$, respectively. The images in these figures are indexed identically to that of Figures 6 through 8. Again, all images within each figure are videoscaled to a common scale. The best restorations with respect to minimum RMS error and $\lambda=1$ for the four nonlinear priors are presented in Table 1, where it is seen that spatial-OR priors can obtain lower RMS error by using greater smoothing factors than the spatial-AND priors. As noted before, the spatial-OR priors preserve step edges better than the spatial-AND priors, as evidenced by observing the step edges of the ring, table and dog-leg in Figures 9 through 11. The signal-AND, spatial-OR restoration is superior to the signal-OR, spatial-OR restoration. This can be seen by comparing the relative uniformity of the piecewise-constant features in the upper-right and center-right frames of Figures 9 through 11.

A comparison of the restorations presented in the upper and center rows of Figures 6 through 11 shows that given noisy data with a piecewise constant underlying structure, and while using a first derivative as a measure of suitability within a neighborhood, best restoration performance is obtained using spatial-OR nonlinear priors. In this context, spatial-OR nonlinear priors allow for much greater smoothing and improved edge restoration compared to the spatial-AND nonlinear priors. The signal-AND prior is slightly superior to the signal-OR prior, since it more accurately 

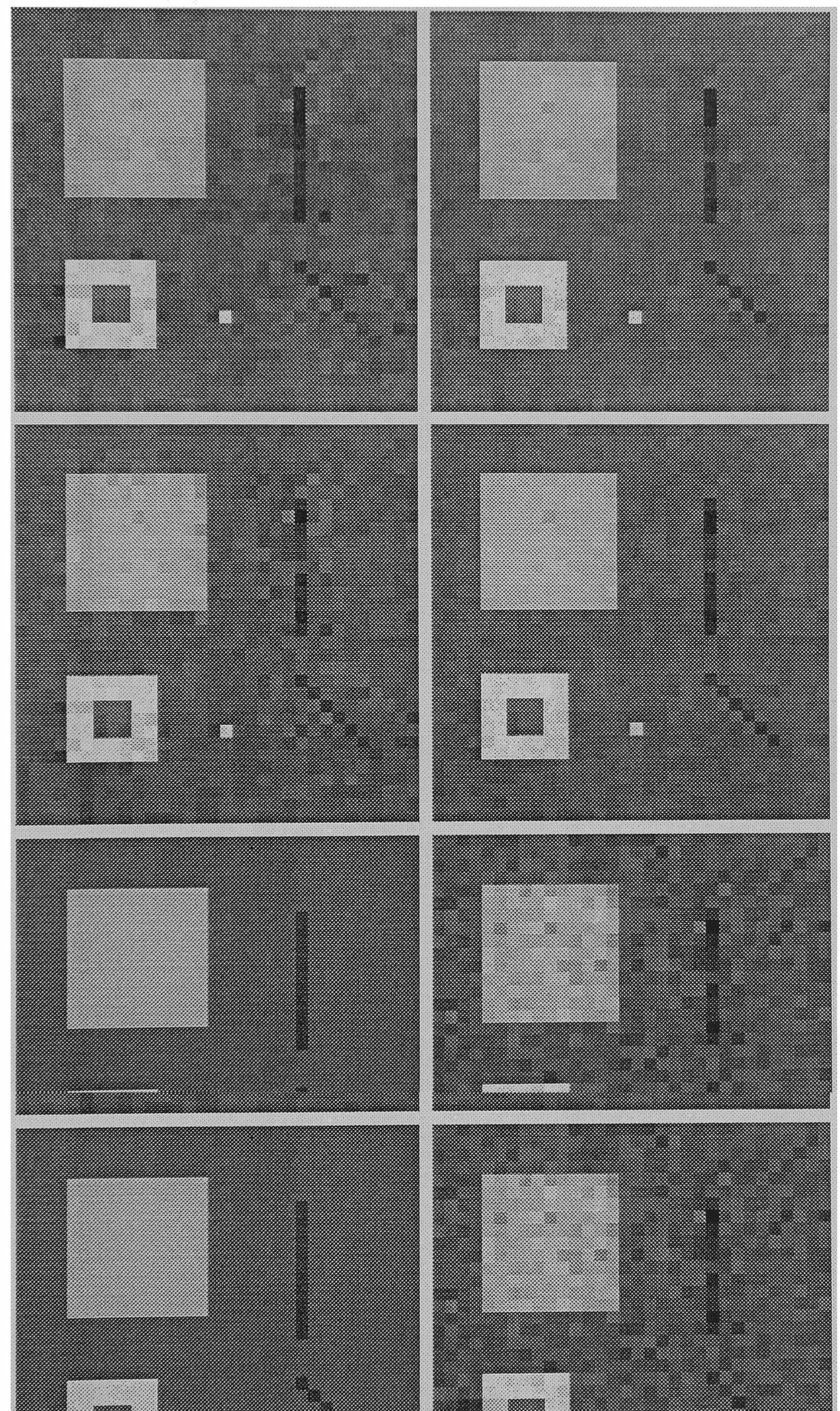

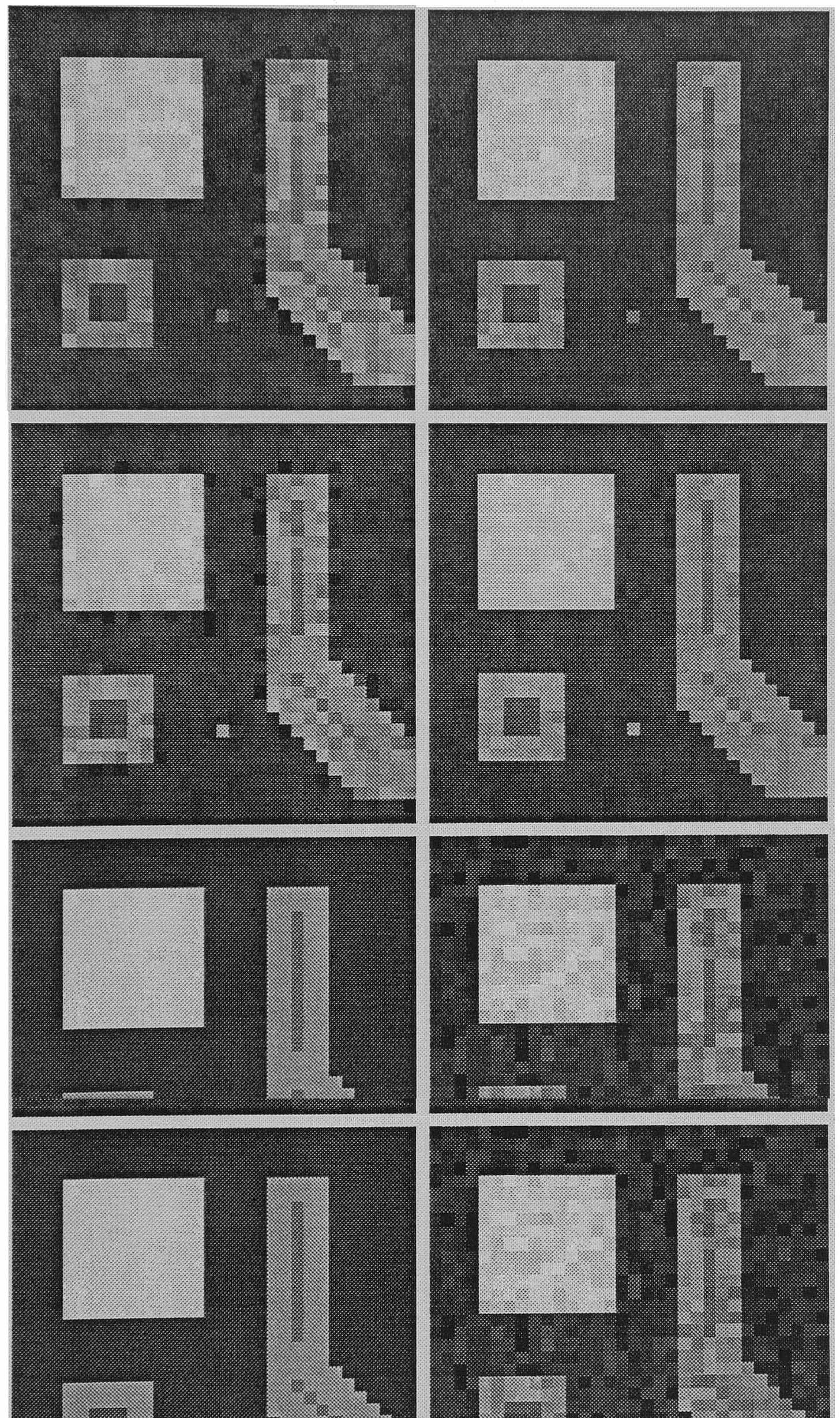

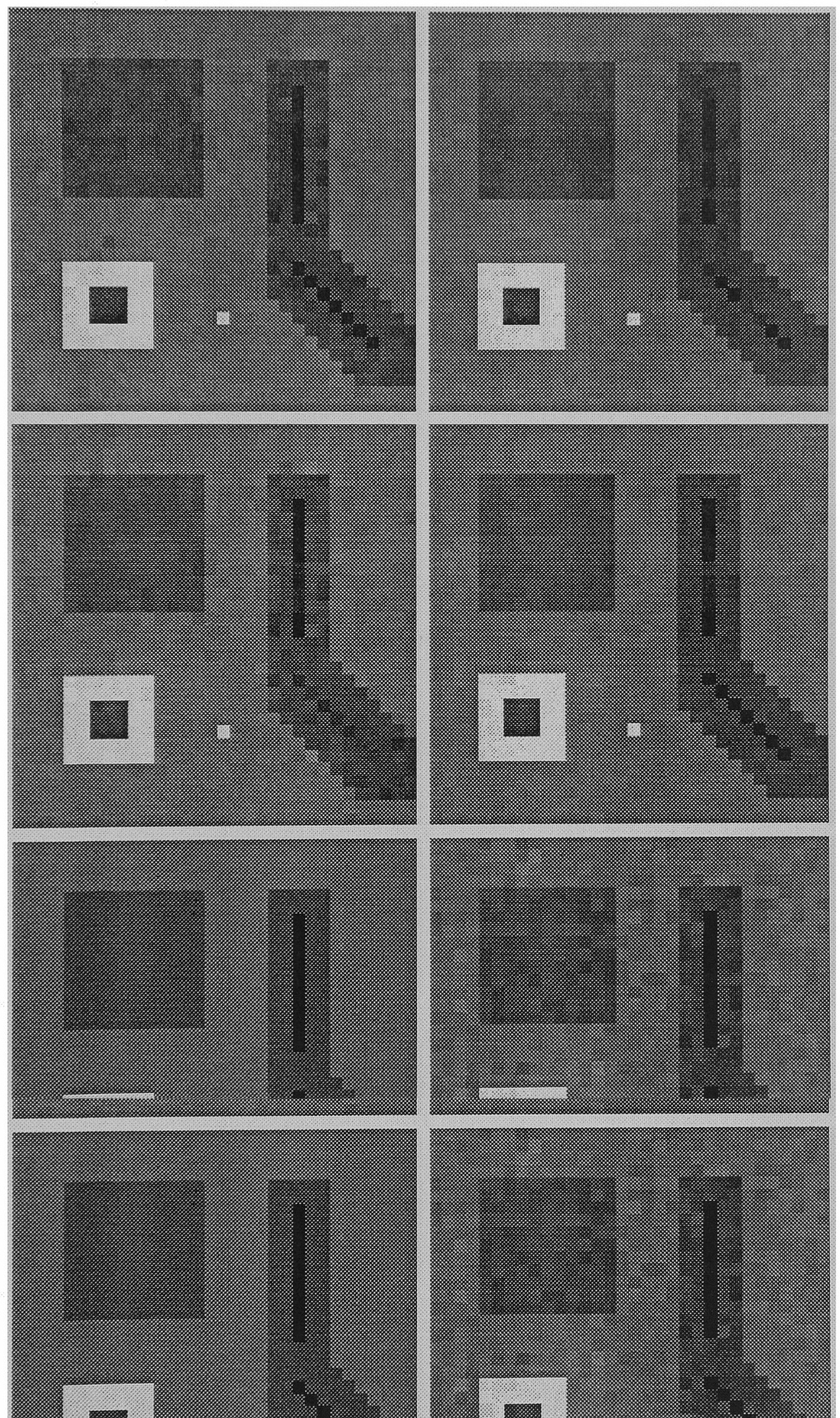

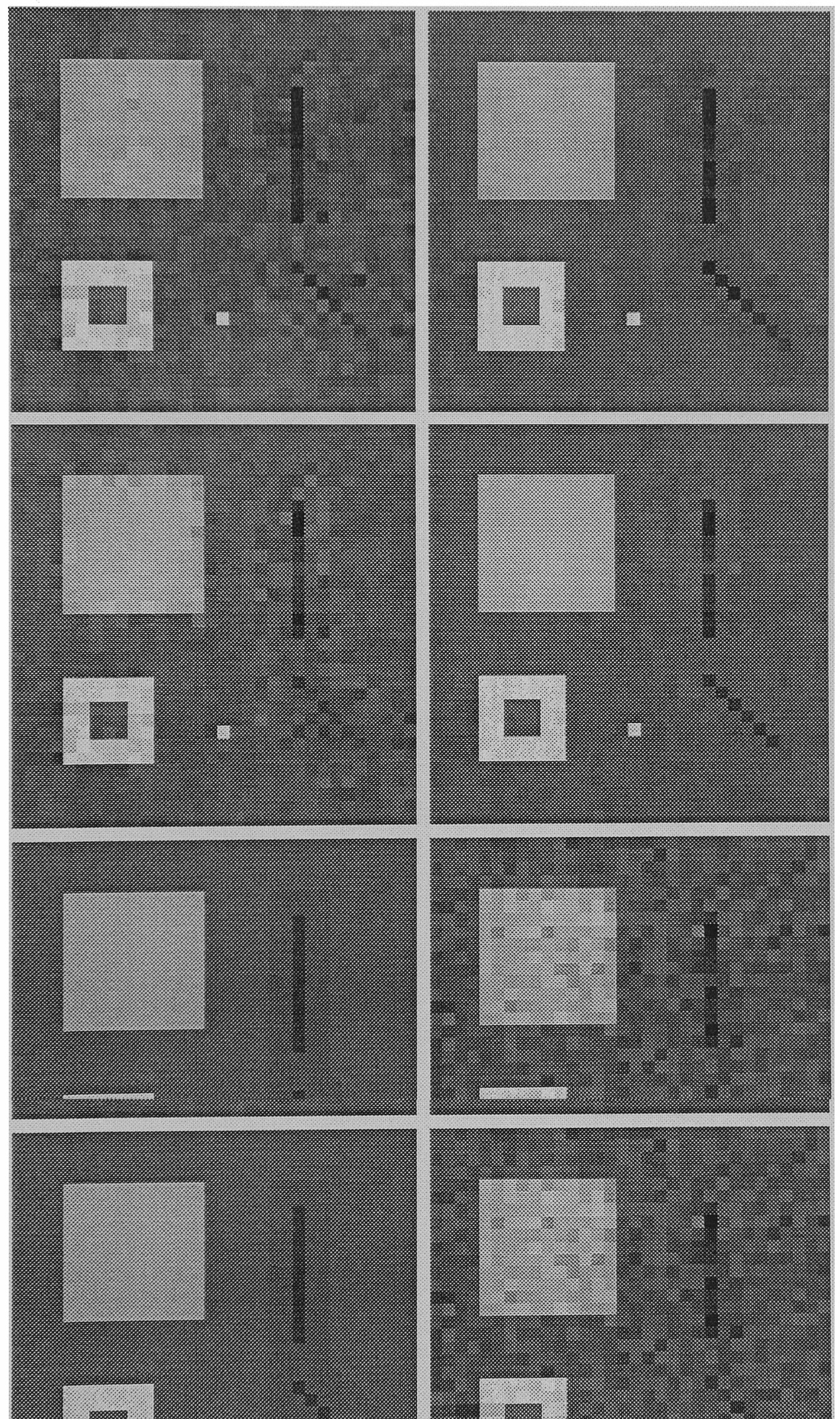

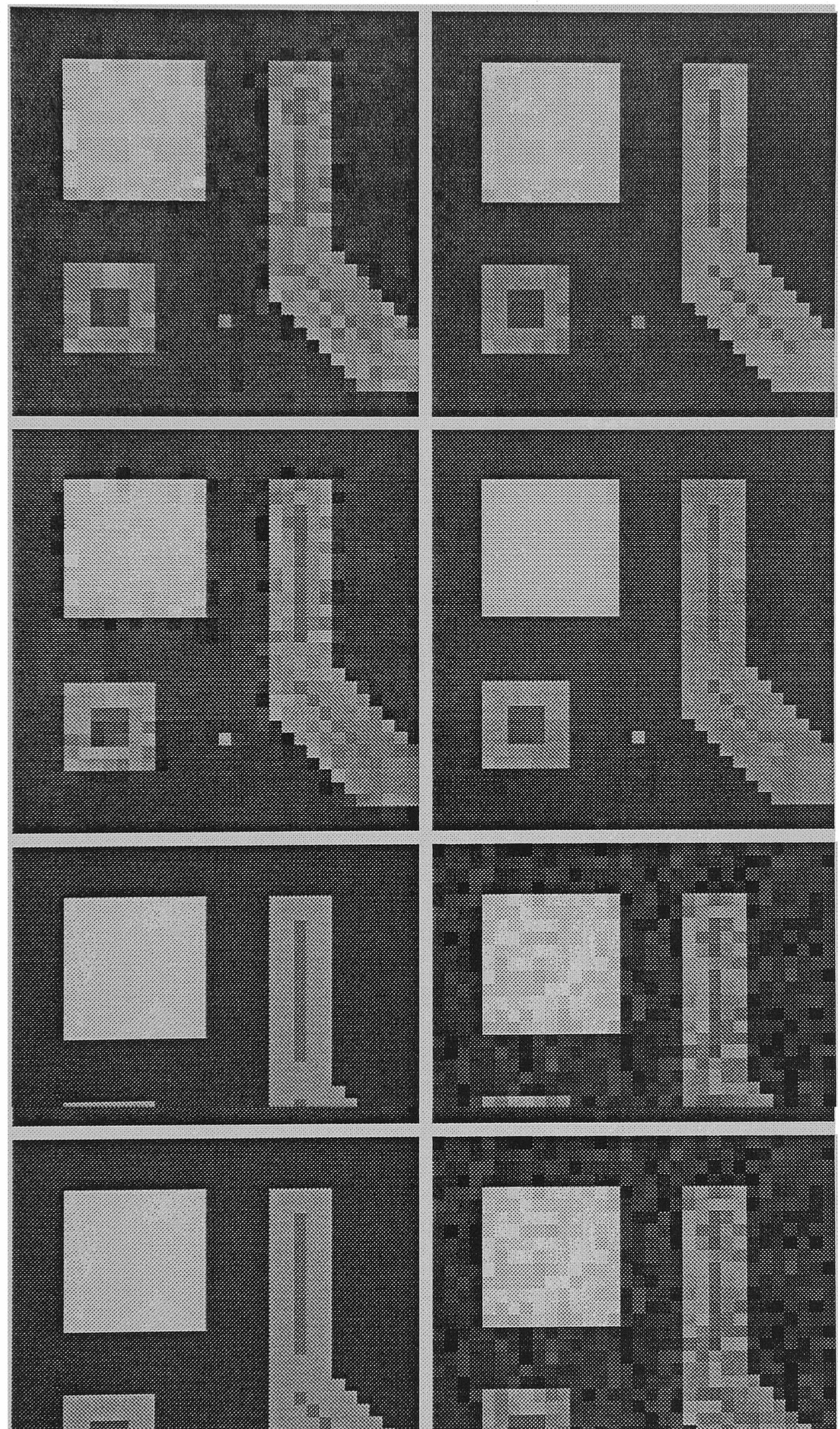

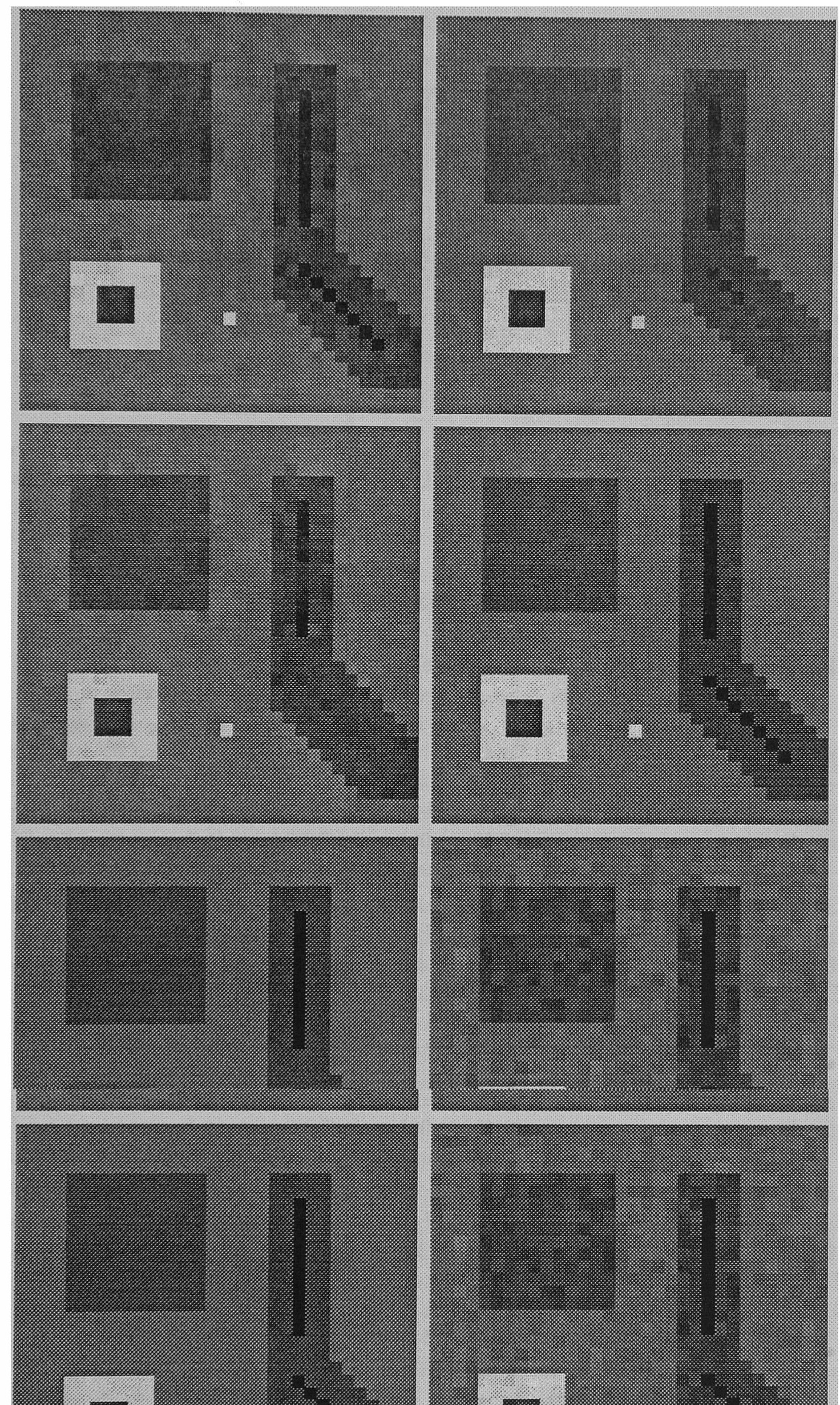


\begin{tabular}{|l|l|l|}
\hline \multicolumn{1}{|c|}{ Prior } & \multicolumn{1}{c|}{$\lambda$} & RMS Error \\
\hline signal-OR, spatial-OR & 4.2 & 10.8 \\
\hline signal-OR, spatial-AND & 0.75 & 15.8 \\
\hline signal-AND, spatial-OR & 7.5 & 9.4 \\
\hline signal-AND, spatial-AND & 3.2 & 15.6 \\
\hline & & \\
\hline signal-OR, spatial-OR & 1.0 & 12.1 \\
\hline signal-OR, spatial-AND & 1.0 & 16.0 \\
\hline signal-AND, spatial-OR & 1.0 & 11.8 \\
\hline signal-AND, spatial-AND & 1.0 & 16.3 \\
\hline
\end{tabular}

Table 1: Minimum RMS Errors for Nonlinear Priors

models the vector interaction of correlated signals within the basis images. The signal-AND prior is particularly well suited to applications where all the basis signals contain features which change simultaneously. The signal-OR prior may be more suitable if one or more basis signals remain unchanged while traversing an image feature.

\subsection{Phantom Data}

Figure 12 shows three acquired images of a plastic bottle containing corn oil acquired by a GE Signa 5.2 scanner with a 1.5 Tesla magnet using spin-echo mode. These images were obtained using three single-echo, single-slice ( 3 millimeter thick) acquisitions using (TE,TR) $=(30,900)$, $(90,900)$ and $(90,1800)$, and they are individually videoscaled to maximize contrast. We acquired two additional $(\mathrm{TE}, \mathrm{TR})=(90,900)$ images in order to compare our MAP results, which were obtained from the images presented in Figure 12, with three averaged data images acquired at (TE,TR) $=$ $(90,900)$. We also obtained three $(\mathrm{TE}, \mathrm{TR})=(30,1800)$ images to compare extrapolations of the ML and MAP restorations with their averaged images.

From the ML restoration, the covariance matrix diagonal elements were determined and used in the MAP algorithm. Figure 13 presents left-to-right the data input into the restoration algorithm, the averaged data and the signal-OR, spatial-OR MAP restoration for $(\mathrm{TE}, \mathrm{TR})=(90,900)$. It is clearly evident that the interpolated MAP restoration is superior to the averaged data. Figure 14 shows the data input into the MAP algorithm (upper-left), the averaged data (upper-right) and the ML (lower-left) and signal-OR, spatial-OR MAP (lower-right) extrapolations for (TE,TR) = $(30,1800)$. The extrapolated MAP restoration is clearly superior to the extrapolated ML restoration, and is reasonably close to the appearance of both the input data and the averaged data.

\subsection{Clinical Data}

All clinical data was acquired by a GE Signa 5.2 scanner with a 1.5 Tesla magnet using spin-echo mode. PD $/ T_{2}$-weighted multi-echo studies were performed to obtain the data in Figures 15 and 16 , 20 and 21 , and 25 and 26 . 

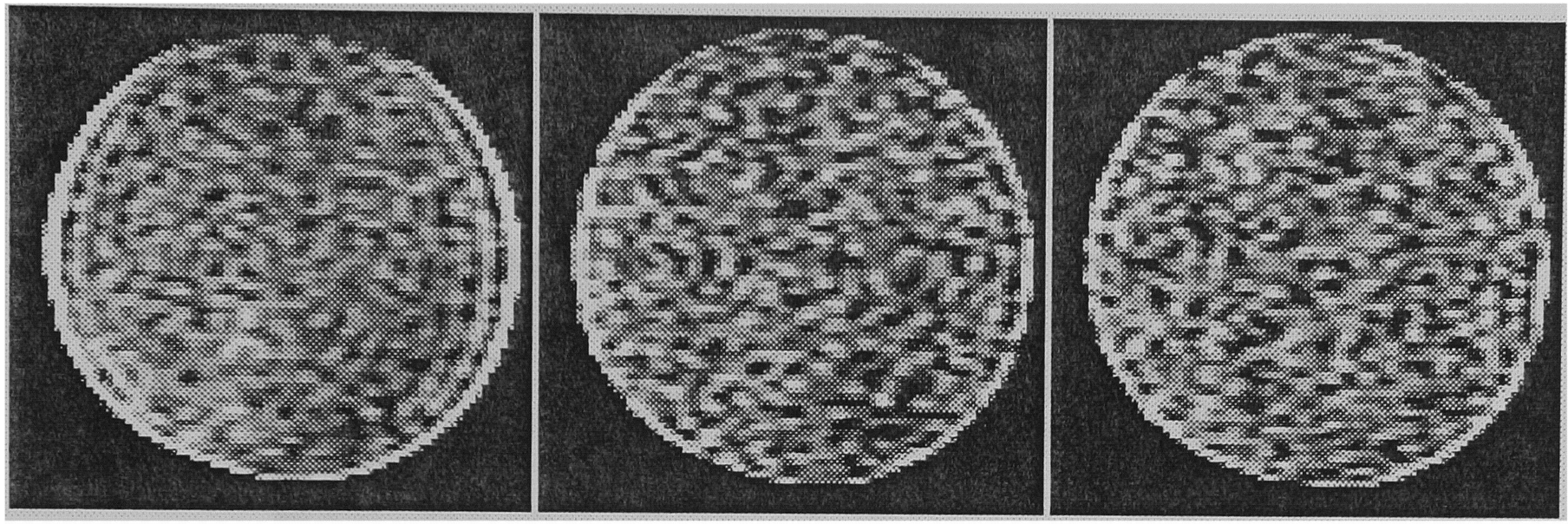

Left: $(\mathrm{TE}, \mathrm{TR})=(30,900)$.

Figure 12: Phantom Data.

Center: $(\mathrm{TE}, \mathrm{TR})=(90,900)$.

Right: $(\mathrm{TE}, \mathrm{TR})=(90,1800)$.
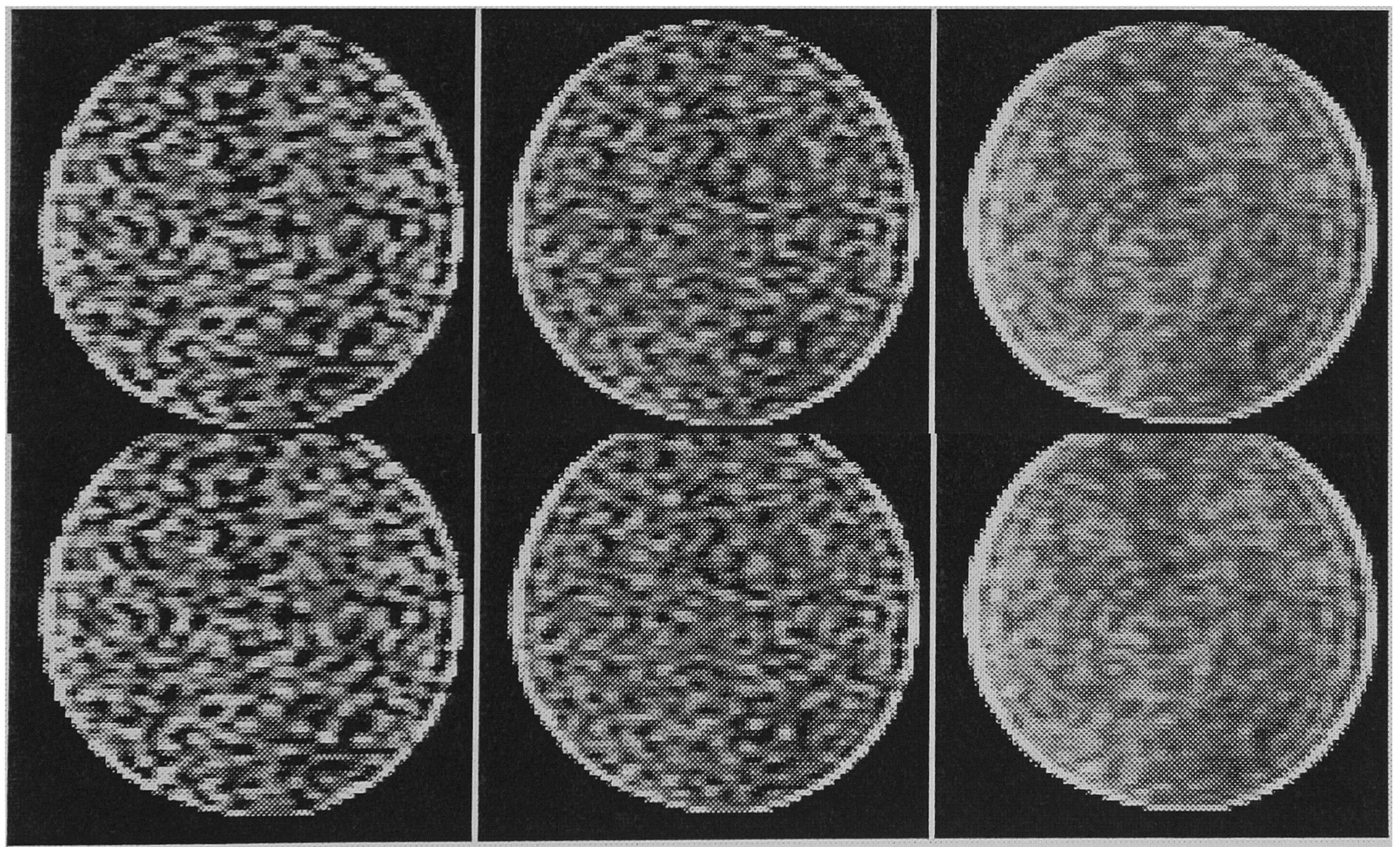


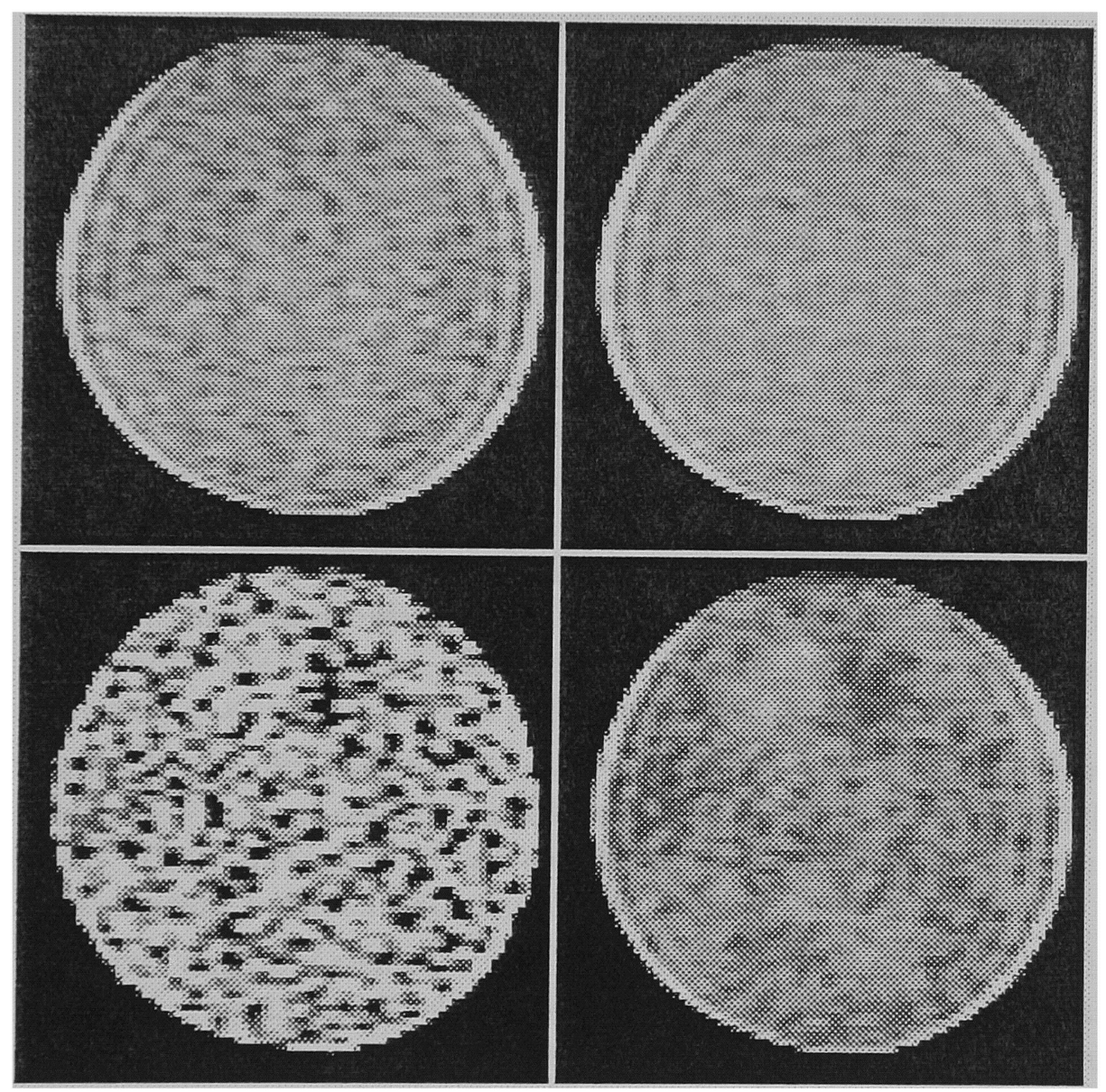

Figure 14: Phantom Results for $(\mathrm{TE}, \mathrm{TR})=(30,1800)$

Upper-Left: Typical Data.

Lower-Left: ML Extrapolation.
Upper-Right: Three Data Average. Lower-Right: MAP Extrapolation.
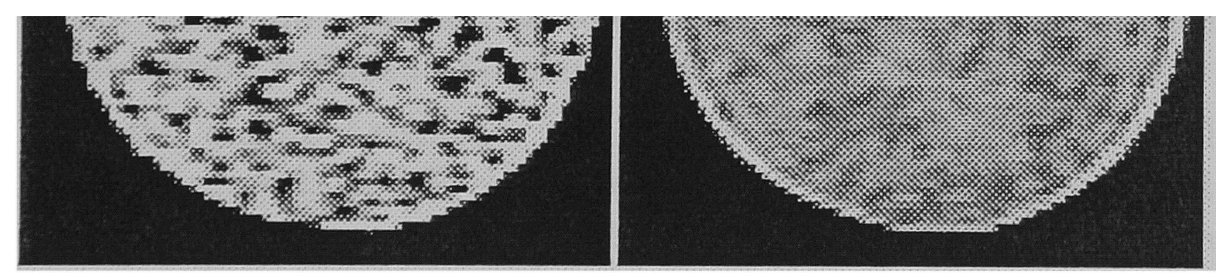

Figure 14: Phantom Results for $(\mathrm{TE}, \mathrm{TR})=(30,1800)$

Upper-Left: Typical Data.

Lower-Left: ML Extrapolation.
Upper-Right: Three Data Average. Lower-Right: MAP Extrapolation. 
Figures 15, 16 and 17 are of a human brain exhibiting some pathological disturbances, and were obtained by two scanning sequences after administration of Gadolinium. The control parameters for these three data images were set at $(\mathrm{TE}, \mathrm{TR})=(32,3000),(90,3000)$ and $(17,417)$, respectively. These images are from one 5 millimeter slice in a multiple slice acquisition using 1 millimeter separation between adjacent slices.

The ML restoration was found for these three data images. Nonlinear restorations were subsequently obtained using the white matter covariance matrix diagonal elements acquired from the ML restoration. Figures 18 and 19 illustrate the $(\mathrm{TE}, \mathrm{TR})=(90,3000)$ restoration results using the prior terms described by Equations 19 and 20, respectively. No ML restorations are shown, since the pixels of three basis images extracted from three valid data points coincide exactly with the data (three equations and three unknowns). While the MAP restorations show a reduction in noise, there is a considerable artifact in the areas of the CSF-filled ventricles for restorations using spatial-OR priors. Within the CSF-filled ventricles, the ML restoration exhibits a large number of nonphysical values for $T_{1}$ and $T_{2}$. Due to the nonlinearity of the physical model, a relatively large change in these nonphysical $T_{1}$ and $T_{2}$ values results in a relatively small increase in the noise term energy when compared to the larger reduction in the prior term energy. The artifact occurs when the stronger interaction of the spatial-OR prior is imposed upon these nonphysical values when they appear within a neighborhood containing realistic physical values. The artifact does not occur with the weaker interaction of the spatial-AND prior, since one single large neighborhood difference allows the restoration to approach the ML case. We suspect these nonphysical values are due to either "interslice interference" [16] [15] [20], fluid flow within the ventricles, or our physical model is too simplistic for multiple-echo acquisitions. If the latter is the case, modification of the physical model would improve both the ML and MAP restored basis images by reducing the quantity of nonphysical observations, particularly within the CSF-filled ventricles.

Figures 20, 21 and 22 were obtained by two scanning sequences of a presumably healthy subject. The control parameters for these three data images were set at $(\mathrm{TE}, \mathrm{TR})=(32,3000),(90,3000)$ and $(17,500)$, respectively. These images are from one 3 millimeter slice in a multiple slice acquisition using 3 millimeter separation between adjacent slices.

The covariance matrix diagonal elements were determined as before from the white matter tissue in the ML restoration. Figures 23 and 24 show the different MAP restoration results of the (TE,TR) $=(32,3000)$ data presented in Figure 20, and are the result of the application of Equations 16 and 18 to our algorithm. Again, there is evidence of algorithm-induced artifact in the CSF-filled ventricles where the spatial-OR priors have been used. All MAP restorations show reduced noise levels when compared to the data and ML restorations.

Figures 25,26 and 27 were acquired using two multi-echo scanning sequences of a presumably healthy subject. The echo and relaxation times were set at $(\mathrm{TE}, \mathrm{TR})=(30,900),(90,900)$ and $(90,1800)$, respectively. These images are one 3 millimeter slice from a multiple slice study using no separation between adjacent slices.

The covariance matrix diagonal elements were determined from the white matter tissue in the ML restoration. Figures 28 and 29 illustrate the restoration outputs due to the application of Equations 18 and 19 , respectively, for the $(\mathrm{TE}, \mathrm{TR})=(90,900)$ setting. While there may be slight evidence of artifact, the spatial-OR restorations are clearly superior with regard to soft tissue differentiation. 


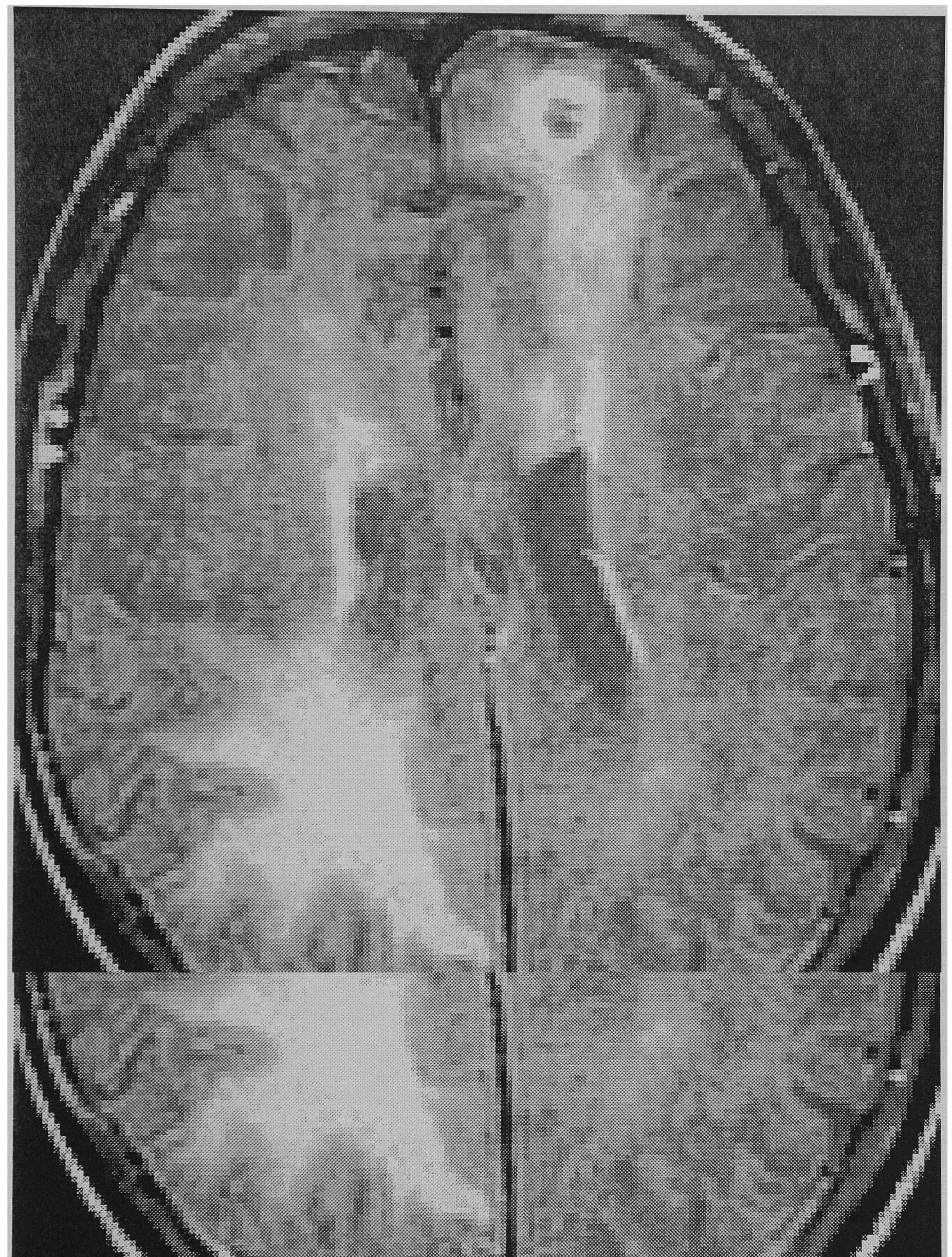




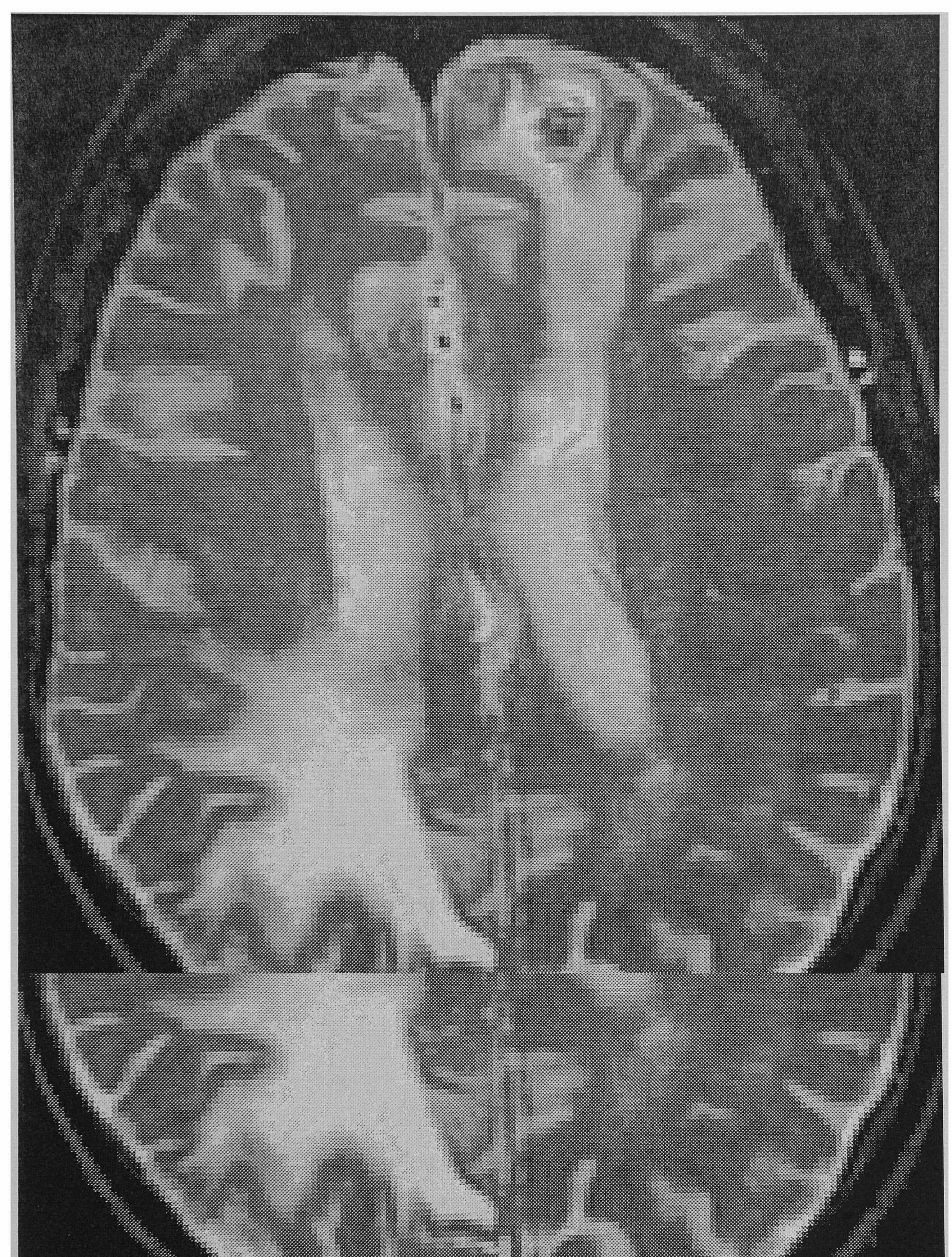




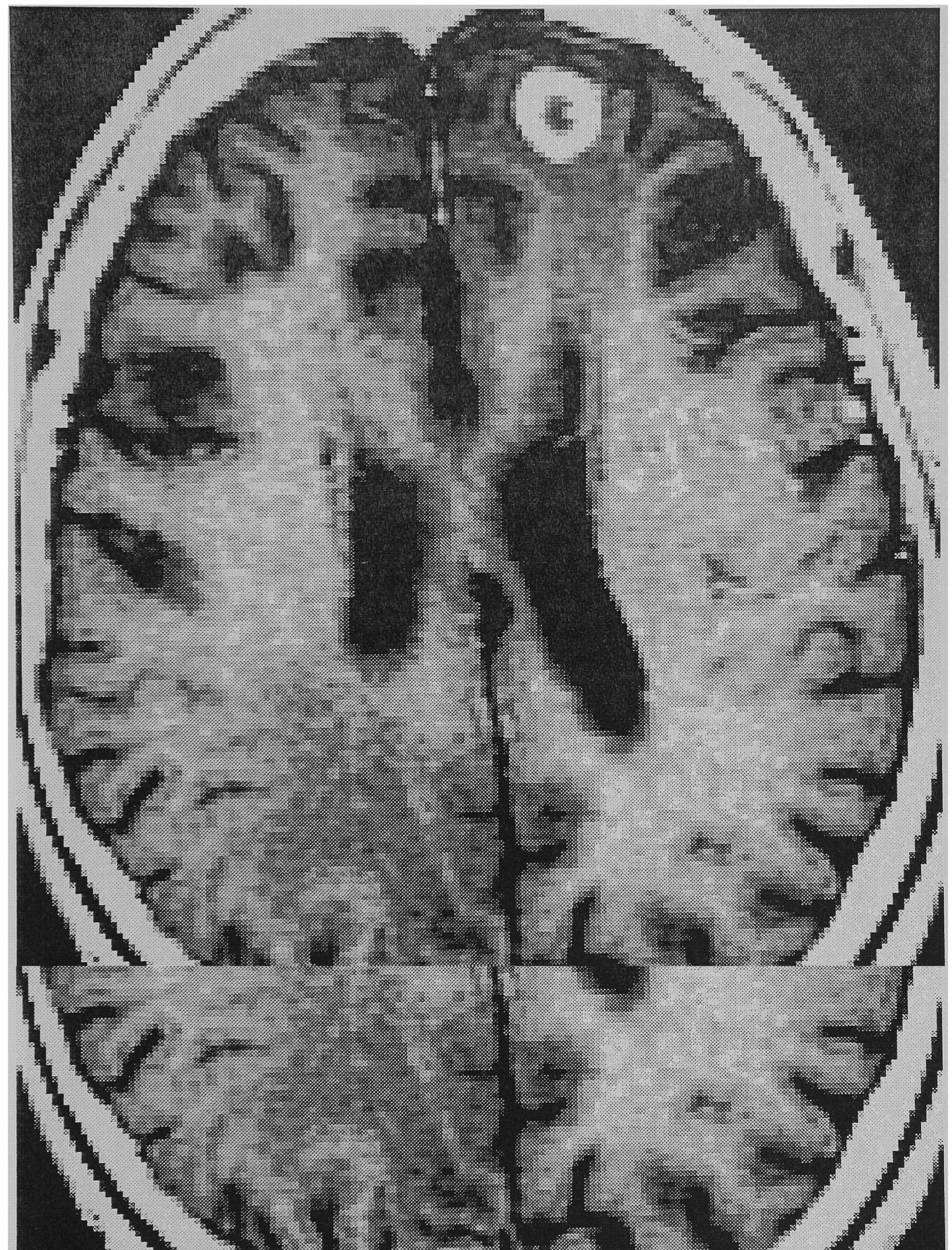




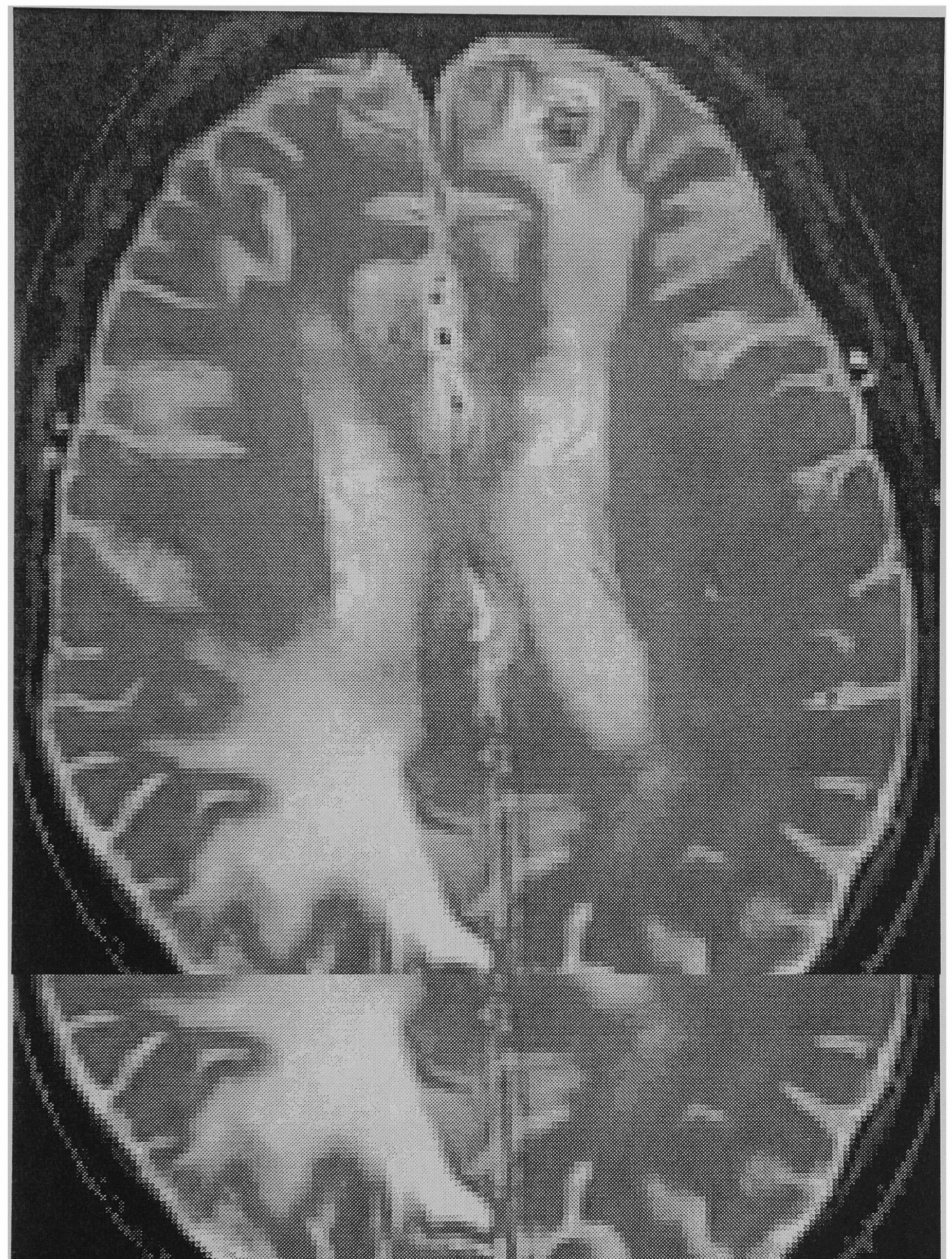




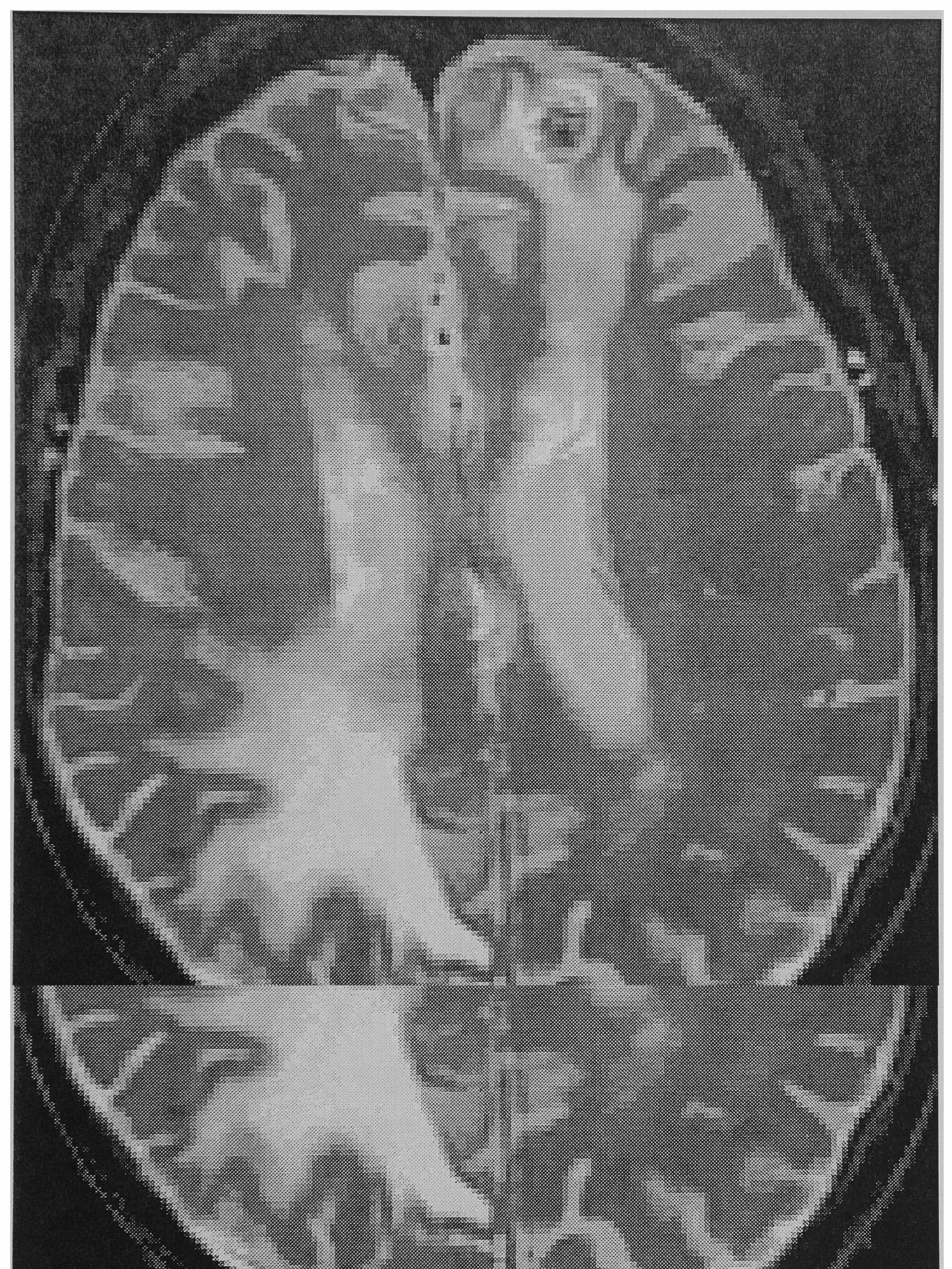




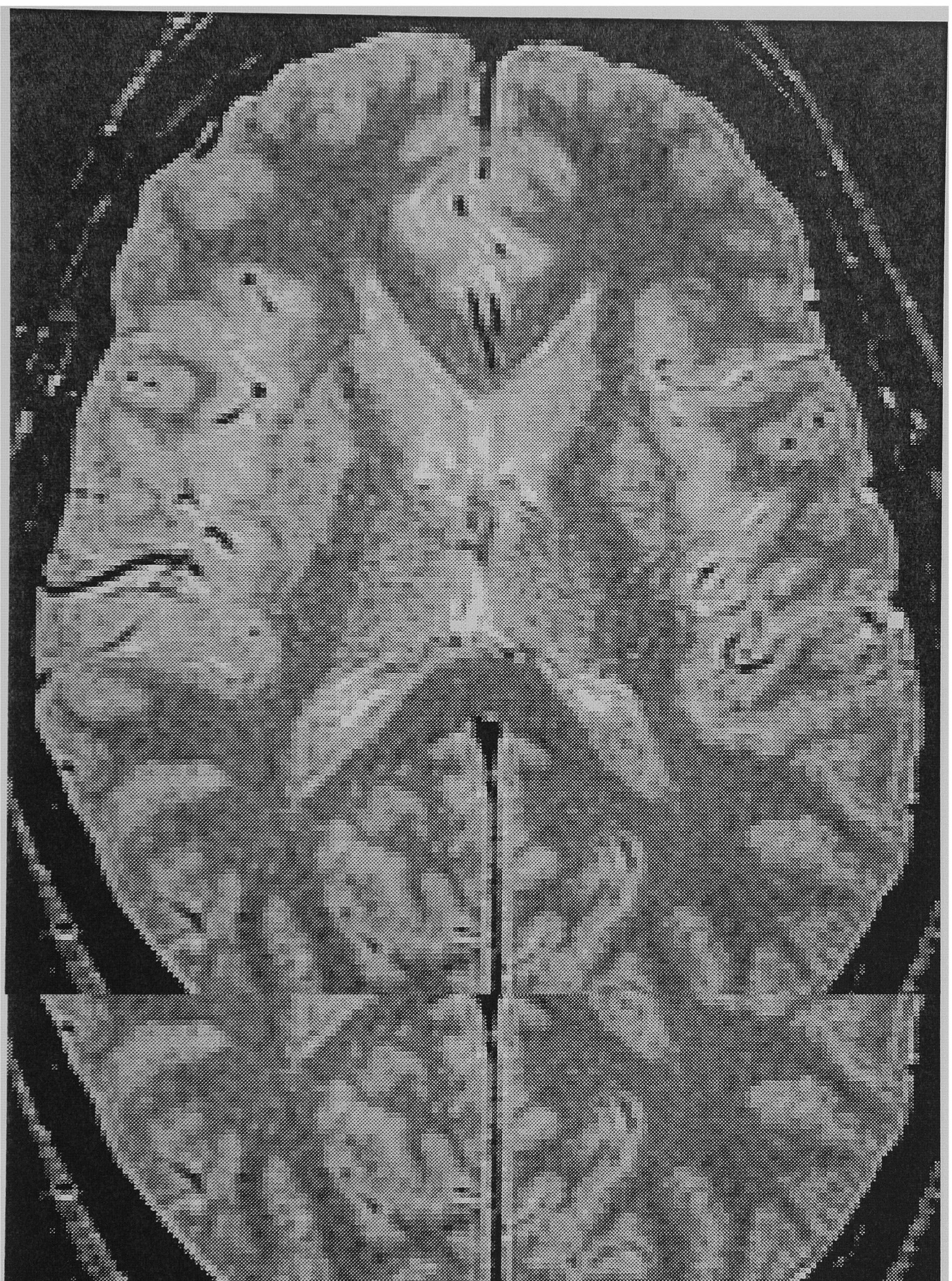




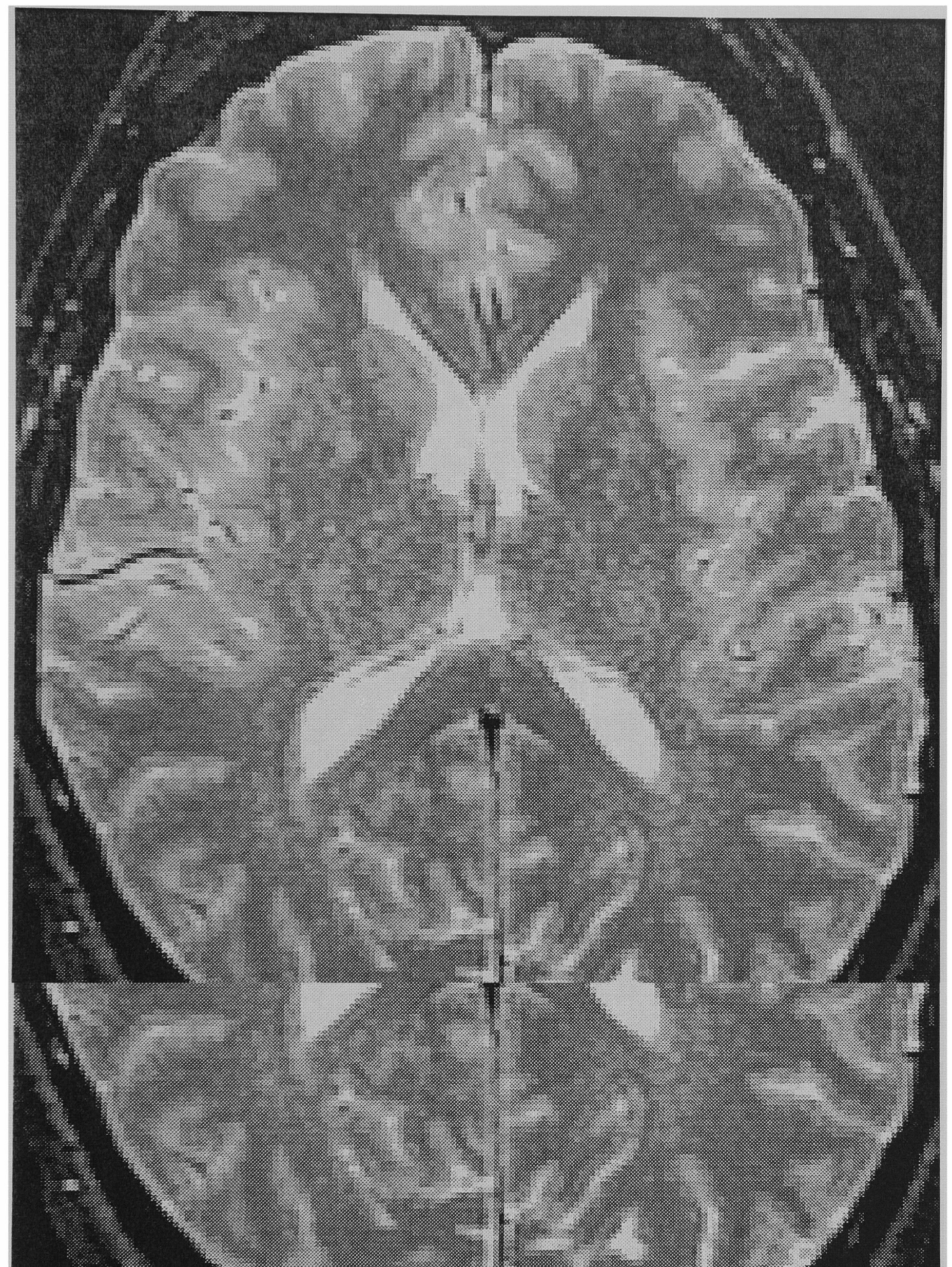




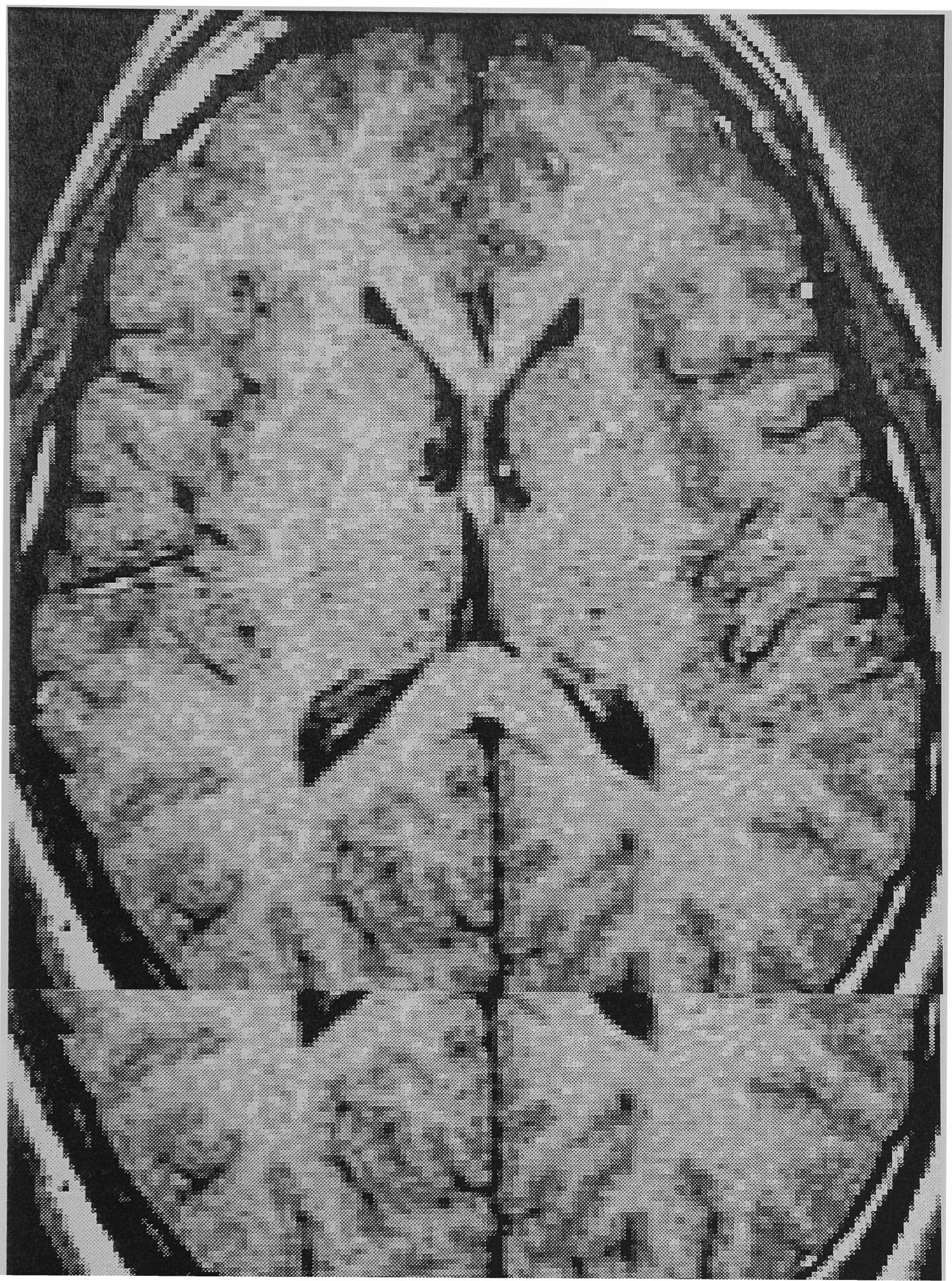




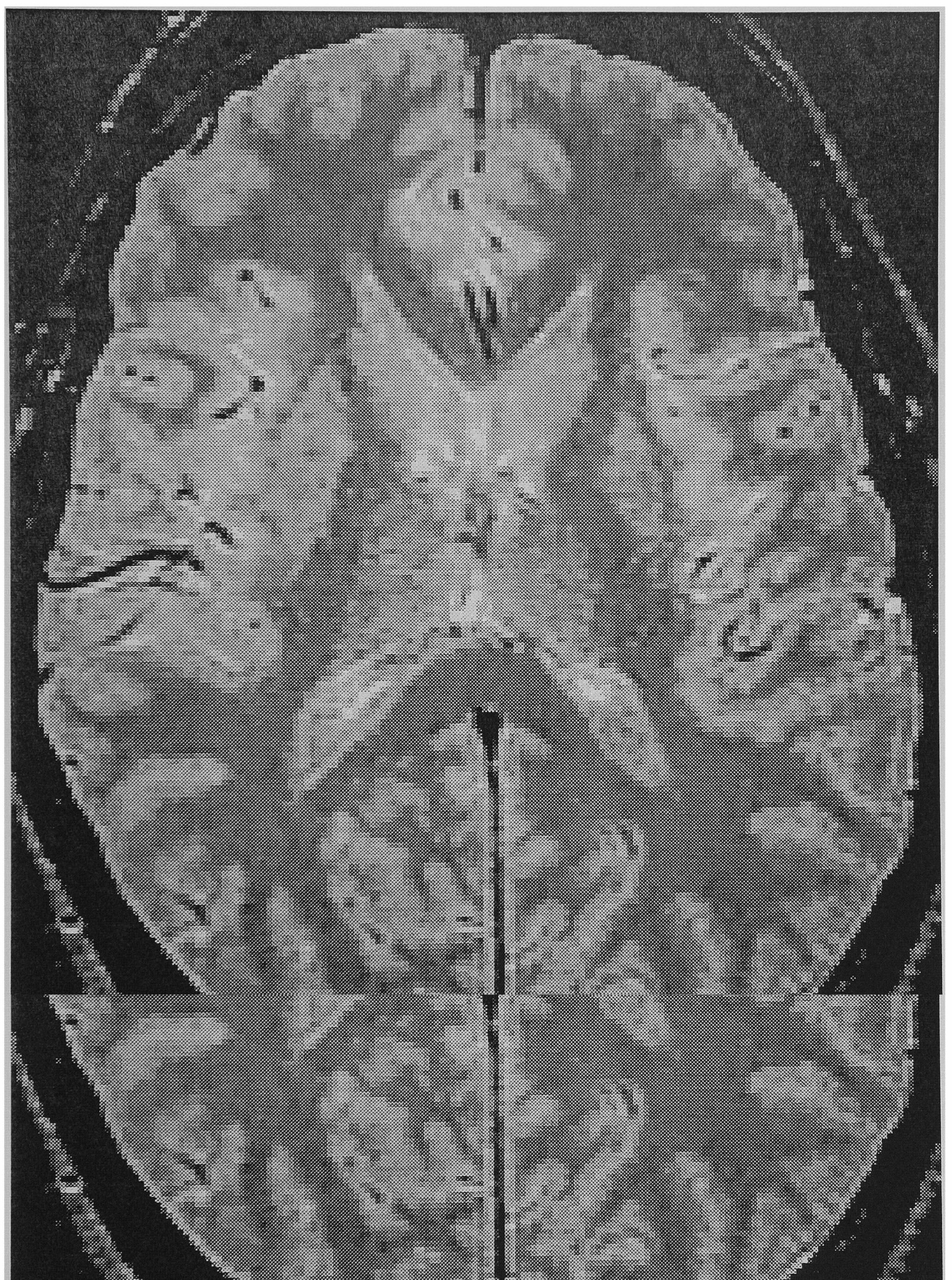




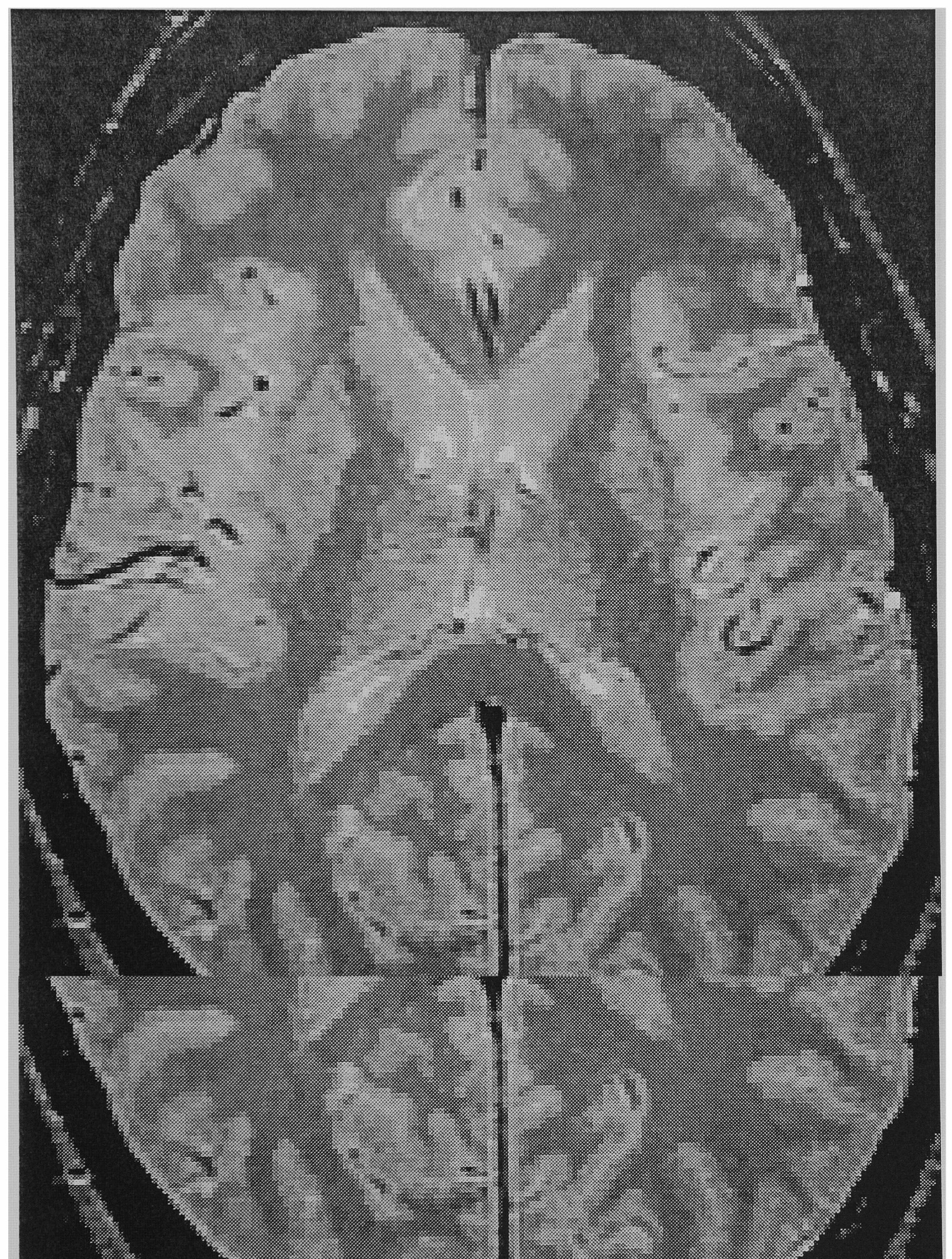




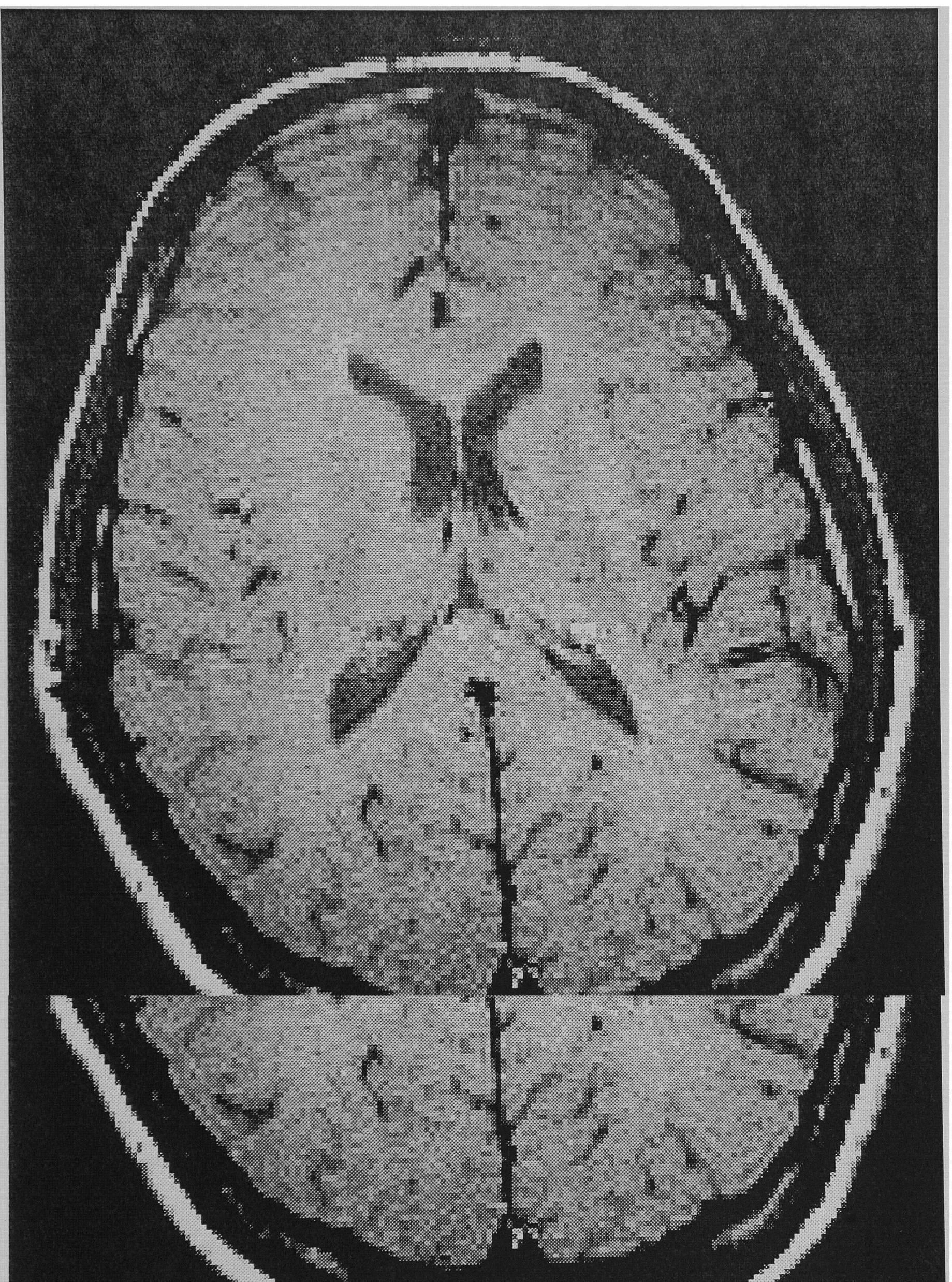




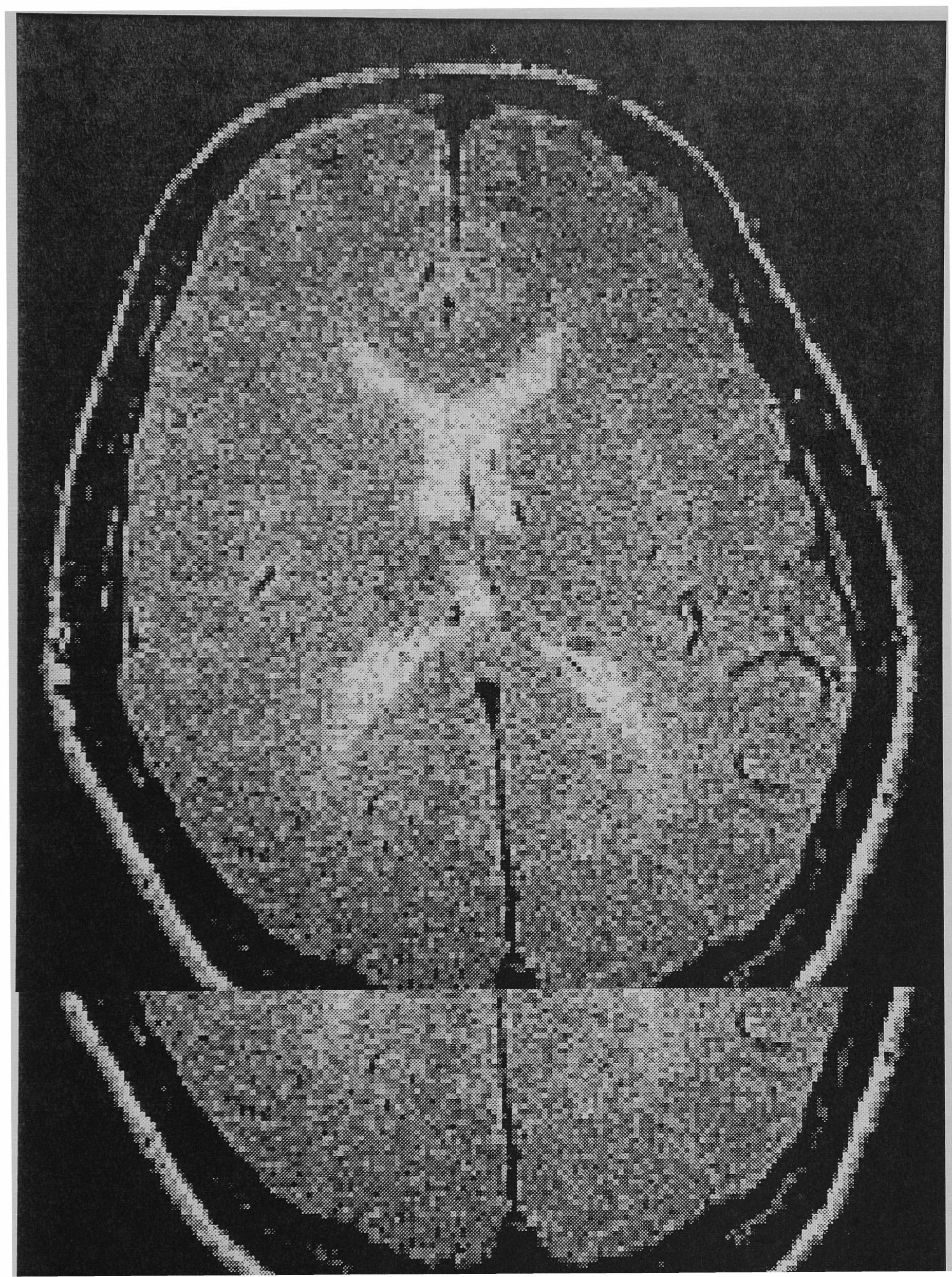




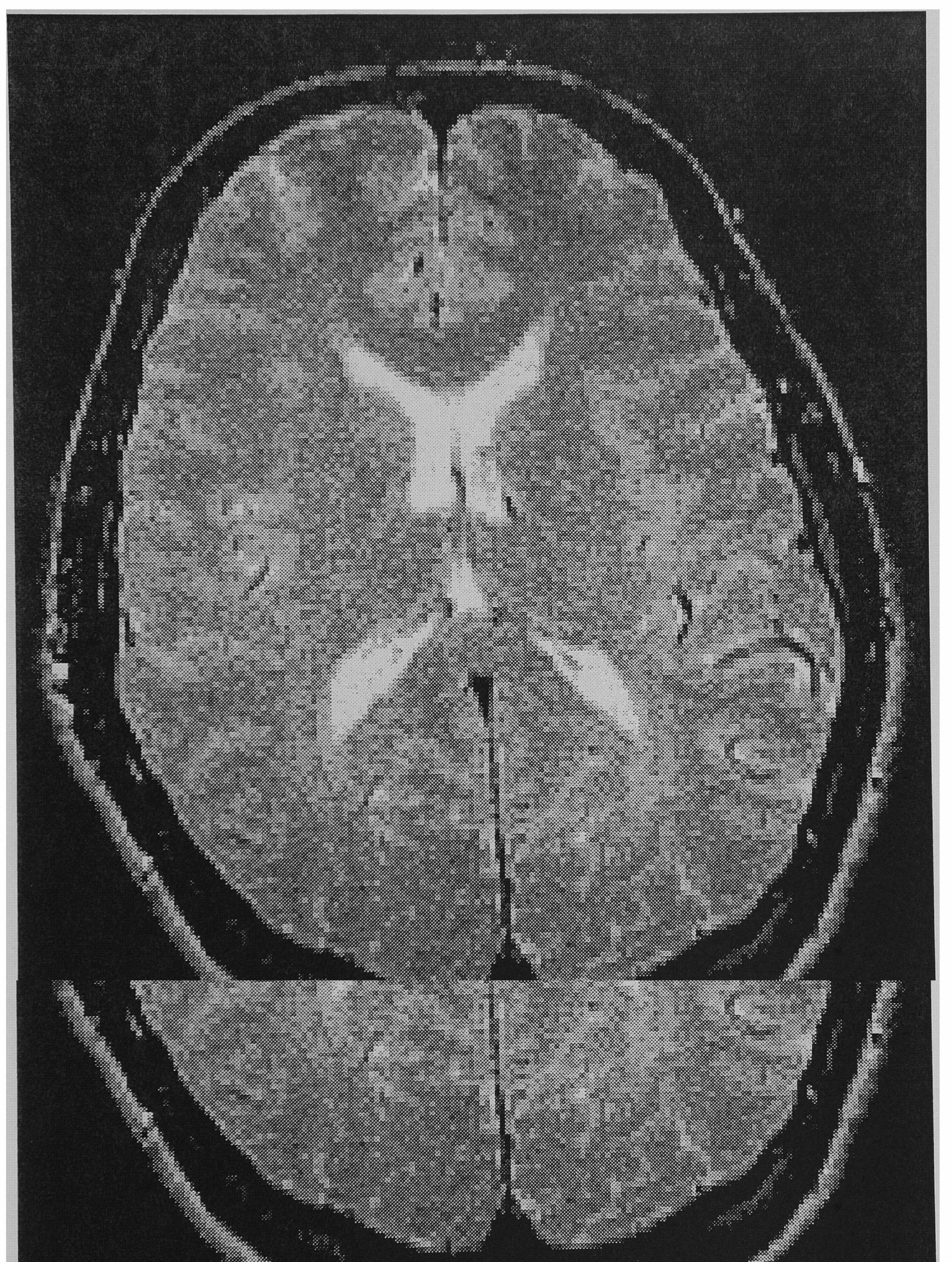




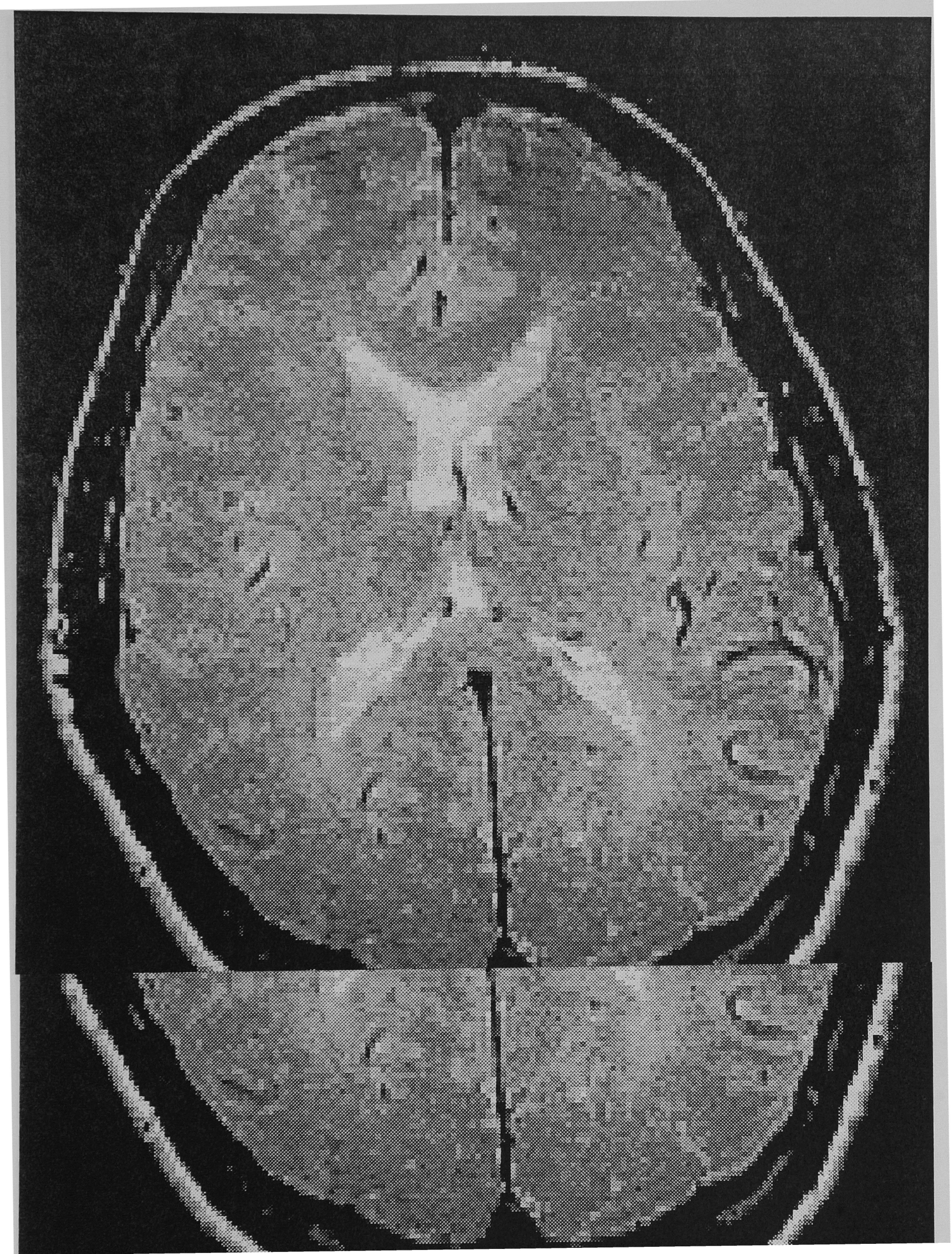




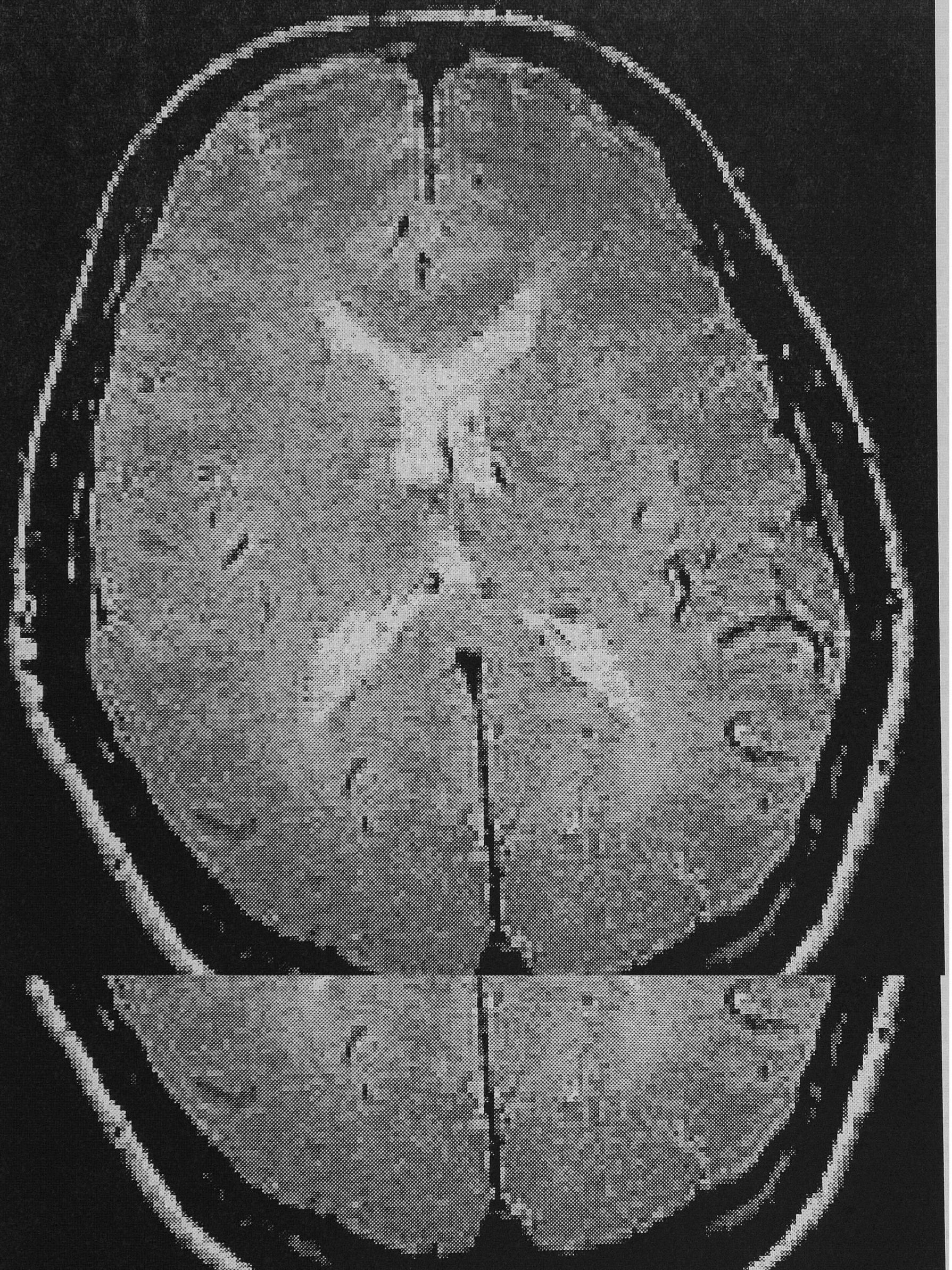




\section{CONCLUSION}

Using acquired data, we have calculated maximum likelihood (ML) and four nonlinearly-smoothed $\rho, T_{2}$ and $T_{1}$ MRI basis image sets, from which we have generated synthetic data restorations. The ML basis images for clinical data show nonphysical values in the CSF-filled ventricles. We suspect these nonphysical values are due to either "interslice interference", fluid flow within the ventricle, or the simplicity of our physical model. If the latter is the case, modification of the physical model would improve both the ML and MAP restored basis images by reducing the quantity of nonphysical observations. The presence of nonphysical values increases the likelihood of the formation of artifacts when spatial-OR priors are used, yet the spatial-OR restorations are clearly superior with regard to soft tissue differentiation. The signal-AND restorations are marginally superior to the signal-OR restorations in the work that we have done since the signal-AND prior more accurately models the interaction of correlated signals.

While additional acquired data might be used to improve the precision of the calculated ML basis images [1], acquiring the additional data with different TR values places a greater time burden on the MR system. When using three data images as input to our algorithm, we have shown that interpolated MAP restorations are superior to the average of three data images at that interpolated (TE,TR) setting. We have also shown that extrapolated MAP restorations, obtained using three relatively low-SNR images as input data, are good approximations to the average of three relatively high-SNR data images at an extrapolated (TE,TR) setting.

In Section 2 we discussed how our MAP restoration algorithm is superior to other methods [5] [4] [22] [19] [18] with regard to noise reduction while simultaneously preserving features. Furthermore, the cited papers do not present algorithms which simultaneously determine proton density, $T_{1}$ and $T_{2}$. Instead they approximate a nonlinear multivariate (3-D) problem into a set of linear $1-\mathrm{D}$ or $2-\mathrm{D}$ problems by a variety of nonlinear transformations on the system of equations, thereby distorting the noise distributions and propagating error into another basis image. They also cannot determine proton density, $T_{2}$ and $T_{1}$ basis signals unless they have acquired two or more images with the same TE, and two or more images with the same TR. Our approach does not have these restrictions. The $\chi^{2}$ approach is a special case of the ML (minimum variance least-squares) approach, and is expected to produce results no better than our ML restorations. $N$ data images may be used to extract proton density, $T_{1}$ and $T_{2}$ in the ML algorithm. If there are $M$ pixels within a single contiguous region of the proton density, $T_{1}$ and $T_{2}$ images possessing similar statistics, then the MAP algorithm uses on the order of $N \times M$ data pixels to develop the restoration value of a single pixel of interest in that region. Approximately $N \times M$ data images would be necessary to produce an ML result with equivalent noise reduction to the MAP result for that single pixel of interest.

Although demonstrated using readily available spin-echo images, this method is easily adaptable to other MR imaging modes by simply changing the physical model. It may be argued that in conventional spin-echo, noise-like artifacts arise primarily from physiological motion and are not (for the most part) from true random noise. However, in Echo Planar (EP) images, acquisition times are so short that motion artifacts are dramatically reduced and to some extent replaced by random noise (with which this algorithm performs best). In EPI using an MTC preparatory pulse, the formulation equation has a form similar to the physical model we have used, and this method should be applicable to removing noise from such images. Investigations are under way.

We have presented a nonlinear-smoothing method, based on a priori knowledge of the local 
characteristics of the tissues of interest, which allows us to reduce noise in the basis images while preserving step edges and other single pixel excursions which a radiologist might find important. Furthermore, we have shown that the restorations generated from these nonlinearly-smoothed basis images are visually superior in noise reduction and edge preservation to those provided by the maximum likelihood basis images. It is in this manner that we have developed a method which obtains images which most clearly differentiate soft tissue types in MRI data. 


\section{ACKNOWLEDGEMENTS}

This work was supported by the U. S. Army Research Office under Contract DAAL03-89-D0003-0004 and by the Center for Communications and Signal Processing, North Carolina State University. We would also like to express our gratitude to Dr. C. A. Hamilton and W. H. Hinson at the Bowman-Gray School of Medicine, Wake Forest University, for providing data images and technical assistance.

\section{References}

[1] C. J. G. Bakker and C. N. De Graff. Precision in calculated $\rho, T_{1}$ and $T_{2}$ images as a function of data analysis method. Magnetic Resonance Imaging, 6:3-8, 1988.

[2] G. L. Bilbro, W. E. Snyder, S. J. Garnier, and J. W. Gault. Mean field annealing: A formalism for constructing GNC-like algorithms. IEEE Transactions on Neural Networks, 3(1):131-138, January 1992.

[3] Griff L. Bilbro and Wesley E. Snyder. Mean field annealing: An application to image noise removal. Journal of Neural Network Computing, 1990.

[4] S. A. Bobman, S. J. Riederer, J. N. Lee, S. A. Suddarth, H. Z. Wang, B. P. Drayer, and J. R. MacFall. Cerebral magnetic resonance image synthesis. American Journal of Neuroradiology, 6:265-269, March/April 1985.

[5] S. A. Bobman, S. J. Riederer, J. N. Lee, S. A. Suddarth, H. Z. Wang, and J. R. MacFall. Synthesized MR images: Comparison with acquired images. Radiology, 155(3):731-738, 1985.

[6] S. A. Bobman, S. J. Riederer, J. N. Lee, T. Tasciyan, F. Farzaneh, and H. Z. Wang. Pulse sequence extrapolation with MR image synthesis. Radiology, 159:253-258, 1986.

[7] T. Brosnan, G. Wright, D. Nishimura, Q. Cao, A. Macovski, and F. G. Sommer. Noise reduction in magnetic resonance imaging. Magnetic Resonance in Medicine, 8:394-409, 1988.

[8] S. J. Garnier, G. L. Bilbro, J. W. Gault, and W. E. Snyder. Magnetic resonance image restoration. Journal of Mathematical Imaging and Vision, 1994. To appear.

[9] D. Geman and S. Geman. Stochastic relaxation, Gibbs distributions, and the Bayesian restoration of images. IEEE Transactions on Pattern Analysis and Machine Intelligence, PAMI6(6):721-741, November 1984.

[10] G. Gerig, O. Kuebler, R. Kikinis, and F. A. Jolesz. Nonlinear anisotropic filtering of mri data. IEEE Transactions on Medical Imaging, 1992.

[11] F. Godtliebsen. Noise reduction using markov random field. Journal of Magnetic Resonance, 92:102-114, 1991.

[12] R. Graumann, H. Fischer, and A. Oppelt. A new pulse sequence for determining $T_{1}$ and $T_{2}$ simultaneously. Medical Physics, 13(5):644-647, September/October 1986. 
[13] Y. S. Han and W. E. Snyder. Multi-variate MR image restoration using vector mean field annealing. In SPIE Proceedings Volume 1768: Mathematical Methods in Medical Imaging, 1992, San Diego, California, July 1992. SPIE's 1992 International Symposium on Optical Applied Science and Engineering.

[14] X. Hu, V. Johnson, W. Wong, and C. Chen. Bayesian image processing in magnetic resonance imaging. Magnetic Resonance Imaging, 9:611-620, 1991.

[15] M. Just, H. P. Higer, and P. Pfannenstiel. Errors in $T_{1}$-determination using multislice technique and Gaussian slice profiles. Magnetic Resonance Imaging, 6:53-56, 1988.

[16] J. B. Kneeland, A. Shimakawa, and F. W. Wehrli. Effect of intersection spacing on MR image contrast and study time. Radiology, 158:819-822, 1986.

[17] J. N. Lee, S. J. Riederer, S. A. Bobman, F. Farzaneh, and H. Z. Wang. Instrumentation for rapid MR image synthesis. Magnetic Resonance in Medicine, 3:33-43, 1986.

[18] J. Liu, A. O. K. Nieminen, and J. L. Koenig. Calculation of $T_{1}, T_{2}$, and proton spin density images in nuclear magnetic resonance imaging. Journal of Magnetic Resonance, 85:95-110, 1989 .

[19] J. R. MacFall, S. J. Riederer, and H. Z. Wang. An analysis of noise propogation in computed $T_{2}$, pseudodensity, and synthetic spin-echo images. Medical Physics, 13(3):285-292, May/June 1986.

[20] S. Majumdar, H. D. Sostman, and J. R. MacFall. Contrast and accuracy of relaxation time measurements in acquired and synthesized multislice magnetic resonance images. Investigative Radiology, 24(2):119-127, February 1989.

[21] D. A. Ortendahl, N. M. Hylton, L. Kaufman, J. C. Watts, L. E. Crooks, C. M. Mills, and D. D. Stark. Analytical tools for magnetic resonance imaging. Radiology, 153(2):479-488, 1984.

[22] R. C. Wright, S. J. Riederer, J. N. Lee, F. Farzaneh, and J. B. De Castro. High-speed techniques for estimating $T_{1}, T_{2}$, and density images. IEEE Transactions on Medical Imaging, MI-6(2):165168, June 1987. 